\title{
Plasmons and Plasmon-Polaritons in Finite Ionic Systems: Toward Soft-Plasmonics of Confined Electrolyte Structures
}

\author{
Janusz Jacak * and Witold Jacak \\ Department of Quantum Technology, Wrocław University of Science and Technology, Wyb. Wyspiańskiego 27, \\ 50-370 Wrocław, Poland; witold.aleksander.jacak@pwr.edu.pl \\ * Correspondence: janusz.jacak@pwr.edu.pl
}

Received: 20 January 2019; Accepted: 11 March 2019; Published: 19 March 2019

check for updates

\begin{abstract}
We address the field of soft plasmonics in finite electrolyte liquid systems ranged by insulating membranes by an analogy to the plasmonics of metallic nanostructures. The confined electrolyte systems can be encountered on a bio-cell organizational level, taking into account that the characteristics of ion plasmons fall to the micrometer size scale instead of the nanometer in metals because of at least three orders of magnitude larger masses of ions in comparison to electrons. The lower density of ions in electrolytes in comparison to density of electrons in metal may also reduce the energy of plasmons by several orders. We provide the fully analytical description of surface and volume plasmons in finite ionic micro-systems allowing for further applications. We next apply the theory of ionic plasmons to plasmon-polaritons in ionic periodic systems. The complete theory of ionic plasmon-polariton kinetics in the chain of micrometer-sized electrolyte spheres, confined by a dielectric membrane, is formulated and solved. The latter theory has next been applied to the explanation of a mysterious and unclear (for several dozen of years) problem of so-called saltatory conduction of the action potential in myelinated axons of nerve cells. Contrary to conventional models of nerve signaling, the plasmon-polariton model pretty well fits to the queer properties of the saltatory conduction. Moreover, the presented application of soft plasmonics to signaling in periodically myelinated axons may allow for identification of a different role in information processing of the white and gray matters in brain and spinal cord. We have outlined some perspectives to utilize the difference between the electricity of myelinated and non-myelinated nerve cells in brain to develop the topological concept of the memory functioning. The proposed ionic plasmon-polariton model of the saltatory conduction differently recognizes the role of the insulating myelin than previously was thought which may be helpful in the development of a better understanding of the demyelination diseases.
\end{abstract}

Keywords: ion plasmons; confined electrolytes; ion plasmon-polaritons; soft-plasmonics; plasmon-kinetics in neurons; saltatory conduction; myelinated axons; braids of neurons; topological model of the mind

\section{Introduction}

In view of plasmons and plasmon-polaritons in metallic nanostructures one can question the similar charge fluctuations in other electrical systems like electrolytes. A challenging problem which we will discuss here is the possibility of occurrence of similar plasmon effects with ionic carriers instead of electrons. Many finite ionic system in the form of closed by membranes electrolyte systems can be encountered in biological structures and the question arises up to which step the plasmonic phenomena would play the role in them and whether the radiative properties of plasmon fluctuation would also be so significant in ionic system as in metals were. It is reasonable that ionic plasmon 
effects would be placed in other regions of energy and wavelength scale in comparison to metallic systems. One can expect that the energy of ionic plasmons is much lower than of electronic plasmons in metals and the typical size of plasmonic finite systems is of micrometer scale. Such a soft plasmonics would be linked with the functionality of biological systems where the electricity is rather of ionic than of electronic character. The cell signaling, membrane transfer or nerve cell conductivity would serve as examples.

For a theoretical model we will adopt for ions, as far as possible, the analogy to metallic nanospheres with plasmon excitations. The ionic systems are much more complicated in comparison to a metal crystal structure with free electrons. Therefore, the identification of appropriate simplifications of the approach to ionic systems is of primary significance. The model must be, however, efficient in repetition for ions in electrolytes of a plasmon scenario known for metal electrons.

Let us consider the finite spherical ionic system (e.g., a liquid electrolyte artificially ranged with a membrane) and identify the plasmon excitations of ions in this system. We will determine their energies for various parameters of the ionic system with special attention paid to irradiation properties of ionic plasmons.

We will analyze an ionic plasmon-polariton propagation in electrolyte sphere chains with prospective relations to signaling in biological systems. Keeping in mind that metallic nano-chains serve as very efficient wave-guides for electro-magnetic signals in the form of collective surface plasmon excitation of wave-type called plasmon-polaritons, we will try to model the similar phenomenon in ionic sphere chains.

For the initial model we will study the spherical (cf. Figure 1) or prolate spheroidal ionic conducting system with balanced charges in analogy to the jellium model in metal where the positive charge background is static and local fluctuations of electron liquid density, negative (beyond the equilibrium density) or positive (beneath the equilibrium density) on the background of the positive jellium, form plasmons in metallic clusters [1]. In the case of an electrolyte we deal, however, with a different situation-in a binary electrolyte both oppositely charged ions are similarly dynamical as they have similar masses and do not exist here in a rigid jellium.
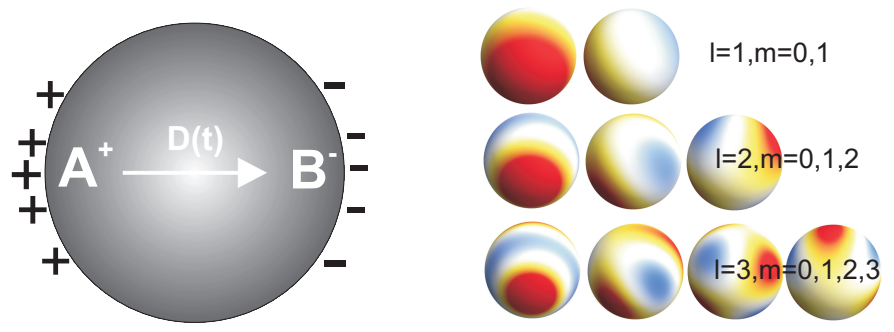

Figure 1. Dipole $\mathbf{D}(\mathbf{t})$ creation in a single sphere by the simplest surface plasmon oscillations (left); examples of surface plasmon charge distribution with various multiplicity $l, m$-different colors indicate distinct values of local charge density from negative to positive ones (right).

In the present review we describe the theory of ion plasmons (surface and volume ones) in finite electrolytes confined by membranes. The spherical shape and symmetry of the electrolyte system is assumed to allow far-reaching analytical formulation taking advantage of properties of spherical functions (Figure 1). The generalization to an arbitrary shape of the finite system is obvious as in the case of metal nanoparticles though it requires an application of the numerical methods. Next, we analyze the radiative properties of ionic plasmons which are essential for plasmon-polariton kinetics in linear ordered arrays of plasmonic components. This analysis allows for the definition and investigation of ionic plasmon-polaritons in linear periodically arranged electrolyte systems in analogy to plasmons-polaritons in metallic nano-chains. The most interesting application of this part of the theory is the original explanation of the mysterious mechanism of the so-called saltatory conduction in myelinated axons. The unknown mechanism of the saltatory conduction phenomenon observed in nerve cell in the peripheral nervous system and in the white matter of 
the central nervous systems-essential for body functioning-clearly goes beyond the explanation ability of conventional models of neuron electricity (the so-called cable theory satisfactory explaining signaling in non-myelinated axons and in dendrites in the gray matter of the central nervous system). Thus the formulated proposition to explain the saltatory conduction by the quite different physical effect-the ionic plasmon-polariton kinetics is the breakthrough idea which might solve the saltatory conduction problem in electro-physiology with important consequences, like a better understanding of the role of the myelin in nerve signaling (the deficiency in the myelin coverage causes severe disorder like Multiple Sclerosis). Finally, we outline some ideas of topological organization of the brain functioning taking advantage of the difference in electricity of the gray and white matter in the brain due to exceptional electro-magnetic wave properties of plasmon-polaritons. The latter are immune to electro-magnetic perturbations and are electro-magnetically passive which seems to agree with various observations of myelinated nerve cell properties. The review demonstrates thus the interdisciplinary influence which would be constructive and beneficial in progress of even far problems.

\section{Fluctuations of Charge Density in Electrolyte Spherical Systems}

The problem of how to establish an adequate model for a multi-ionic system in order to grasp essential properties of ionic plasmons is a challenging issue. For a simple two-component ionic system (binary electrolyte) we deal with both sign ion solution, creating an electrolyte with balanced negative and positive total charges. In equilibrium, these charge cancellations are also local, corresponding to a uniform charge distribution of both signs. As we deal with two kinds of carriers, they both would form density fluctuations resulting in violation of the local electric equilibrium. The total compensation of both sign charges requires, however, that any density fluctuation of negative charges must be accompanied by distant (in general), but ideally equivalent positive ion fluctuation. This means that effectively we deal with charge density fluctuations of both ion subsystems with respect to an equilibrium homogeneous neutral state. One can thus consider the local effective fluctuations of, say, negative ions, positive or negative in charge value with respect to the uniform charge distribution assumed as ideally canceled by the positive uniform background-fictitious jellium in analogy to the metal. In this way we can model the two-component ionic system by two single-component ion systems with double jellium of opposite signs. These opposite sign fictitious jellium cancel themselves. However, both oppositely charged ion fluctuations mutually interact. For simplicity, we assume that the absolute values of charges and the masses of opposite ions are the same, but the generalization to different ion characteristics is straightforward. A more complicated problem may arise, however, in ternary electrolytes (or with even higher number of different ion components)—each of such more complicated electrolytes requires separate analysis, which we do not address it here.

To simplify the model according to the above described lines let us consider the spherical shape system with radius $a$ with balanced total charge of both sign ions with uniform equilibrium density distributions $n^{+(-)}(\mathbf{r})=n \Theta(a-r)(\Theta(r)$ is the Heaviside step function, $a$ is the sphere radius), and we assume, for simplicity, the same absolute value of charges of plus- and minus-charged ions. By $m^{+(-)}$ one can denote the mass of positive (negative) ions. They both are of the order of $10^{4-5} m_{e}$, where $m_{e}=9.1 \times 10^{-31} \mathrm{~kg}$ is the mass of the electron. The reduction of the two-component system to two systems with the fictitious jellium is an approximation, but may serve for a tentative recognition of ionic dynamics and the assessment of scales for its quantitative characteristics, at least. The advantage of such an approach is its close analogy to the description of plasmons in metals where the rigid shape of the system has been imposed by the shape of the real jellium-crystal lattice.

Upon the above model assumptions we will consider the ionic carries with the density oscillating around the zero-valued equilibrium density and balanced by the fictitious charged background of opposite-sign ions (the effect of the opposite sign ion simultaneous presence). The electrolyte is the sum of two such subsystems corresponding to both sign ions each with own fictitious jellium. Both these jellium cancel mutually themselves. The uniform charge distribution of both sign ions also cancel themselves mutually and the approximation is the assumption of independence of fluctuations is such 
subsystems. In this way the approximate correspondence to electron liquid in metal within jellium model is established. Hence, the description of fluctuations of local density of electrons in metallic nanosphere can be directly used to model fluctuations of effective ions density, substituting electron mass by the ion mass and the electron charge by the ion charge. The dynamical equation for the charged fluid in the spherical ion system can be thus repeated from the case of metal nanosphere with electrons. The equilibrium density of the effective charged liquid, denoted by $n$, will be treated as a parameter and assumed equal to $n=\eta N_{0}$ where $\eta$ is the molarity of the electrolyte in the sphere and $N_{0}$ is the one-molar electrolyte concentration of ions. The equilibrium density determines the bulk plasmon frequency for the ion system according to the formula, $\omega_{p}^{2}=\frac{4 \pi n q^{2}}{m}$, where $n$ and $m$ are the equilibrium uniform concentration and the mass of ions with the charge $q$. Due to larger $m$ than $m_{e}$ and usually smaller concentration of ions than that for electrons in metals, $\omega_{p}$ can be considerably reduced even by several orders of the magnitude. Note, that for electrons in metals $\hbar \omega_{p} \simeq 10 \mathrm{eV}$ and typically falls into ultra-violet region of radiation with corresponding energy of photons. In the ionic system, the plasmon frequency $\hbar \omega_{p}$ can be much lower and placed in the range of infra-red or even in lower energy part of the electro-magnetic wave spectrum.

\section{Model Definition}

The Hamiltonian for the binary ion system has the form,

$$
\begin{aligned}
\hat{H}_{i o n}= & -\sum_{i=1}^{N^{-}} \frac{\hbar^{2} \nabla_{i}^{2}}{2 m^{-}}-\sum_{j=1}^{N^{+}} \frac{\hbar^{2} \nabla_{j}^{2}}{2 m^{+}}-\sum_{i, j}^{N^{-}, N^{+}} \frac{q^{-} q^{+}}{\varepsilon\left|\mathbf{r}_{i}-\mathbf{r}_{j}\right|} \\
& +\frac{1}{2} \sum_{i, i^{\prime}, i \neq i^{\prime}}^{N^{-}} \frac{\left(q^{-}\right)^{2}}{\varepsilon\left|\mathbf{r}_{i}-\mathbf{r}_{i^{\prime}}\right|}+\frac{1}{2} \sum_{j, j^{\prime} j \neq j^{\prime}}^{N^{+}} \frac{\left(q^{+}\right)^{2}}{\varepsilon\left|\mathbf{r}_{j}-\mathbf{r}_{j^{\prime}}\right|}
\end{aligned}
$$

where $q^{-(+)}, M^{-(+)}, N^{-(+)}$are the charge, the mass and the number of the $-(+)$ions, respectively. To analyze this complicated system we propose the following approximation, assuming, for simplicity, $q^{-}=-q^{+}=q, N^{-}=N^{+}=N, m^{-}=m^{+}=m$. We next add and subtract the same terms as written below,

$$
\begin{aligned}
& \hat{H}_{i o n}=-\sum_{i=1}^{N} \frac{\hbar^{2} \nabla_{i}^{2}}{2 m}-\sum_{j=1}^{N} \frac{\hbar^{2} \nabla_{j}^{2}}{2 m}-\sum_{i, j} \frac{q^{2}}{\varepsilon\left|\mathbf{r}_{i}-\mathbf{r}_{j}\right|}+\frac{1}{2} \sum_{i, i^{\prime}, i \neq i^{\prime}}^{N} \frac{q^{2}}{\varepsilon\left|\mathbf{r}_{i}-\mathbf{r}_{i^{\prime}}\right|}+\frac{1}{2} \sum_{j, j^{\prime} j \neq j^{\prime}}^{N} \frac{q^{2}}{\varepsilon\left|\mathbf{r}_{j}-\mathbf{r}_{j^{\prime}}\right|} \\
& -q^{2} \sum_{j} \int \frac{n(\mathbf{r}) d^{3} \mathbf{r}}{\varepsilon\left|\mathbf{r}_{j}-\mathbf{r}\right|}-q^{2} \sum_{i} \int \frac{n(\mathbf{r}) d^{3} \mathbf{r}}{\varepsilon\left|\mathbf{r}_{i}-\mathbf{r}\right|}+q^{2} \sum_{j} \int \frac{n(\mathbf{r}) d^{3} \mathbf{r}}{\varepsilon\left|\mathbf{r}_{j}-\mathbf{r}\right|}+q^{2} \sum_{i} \int \frac{n(\mathbf{r}) d^{3} \mathbf{r}}{\varepsilon\left|\mathbf{r}_{i}-\mathbf{r}\right|}
\end{aligned}
$$

where we have introduced formally the fictitious jellium of the spherical shape for both types of ions, with the density $n$ ideally compensating opposite charges of uniformly distributed ions, $n(\mathbf{r})=$ $n \Theta(a-r), a$ is the sphere radius (the positive with the negative jellium mutually cancel themselves). Assuming that,

$$
q^{2} \sum_{j} \int \frac{n(\mathbf{r}) d^{3} \mathbf{r}}{\varepsilon\left|\mathbf{r}_{j}-\mathbf{r}\right|}+q^{2} \sum_{i} \int \frac{n(\mathbf{r}) d^{3} \mathbf{r}}{\varepsilon\left|\mathbf{r}_{i}-\mathbf{r}\right|}-\sum_{i, j} \frac{q^{2}}{\varepsilon\left|\mathbf{r}_{i}-\mathbf{r}_{j}\right|} \simeq 0
$$

which is fulfilled for not too strong ion fluctuations beyond the uniform distribution (in dilute ion systems), we can separate the Hamiltonian into the sum, $\hat{H}_{\text {ions }}=\hat{H}^{-}+\hat{H}^{+}$, where,

$$
\hat{H}^{-(+)}=\sum_{j}\left[-\frac{\hbar^{2} \nabla_{j}^{2}}{2 m}-q^{2} \int \frac{n(\mathbf{r}) d^{3} \mathbf{r}}{\varepsilon\left|\mathbf{r}_{j}-\mathbf{r}\right|}\right]+\frac{1}{2} \sum_{j \neq j^{\prime}} \frac{q^{2}}{\varepsilon\left|\mathbf{r}_{j}-\mathbf{r}_{j^{\prime}}\right|} .
$$

The latter term in r.h.s. of Equation (4) corresponds to the interaction between ions of the same sign, whereas the second term in the first sum describes the interaction of these ions with the effective jellium (of opposite sign), $\varepsilon$ is the dielectric permittivity of the electrolyte medium. The confined electrolyte system can be shaped by appropriately formed membrane, as frequently happens in biological systems. 
Because of separation of the Hamiltonian (1) one can consider the single Hamiltonian (4). The second one is analogous. The ion wave function corresponding to Hamiltonian (4) we will denote by $\Psi_{\text {ion }}(t)$.

The form of Hamiltonian (4) allows for the repetition of its further discussion along the scheme applied to electrons in metals [1], which we will recall below, for the sake of completeness. A local density of the chosen type ions can be written, in analogy to semiclassical Pines-Bohm random phase approximation (RPA) approach to electrons in metal [2], in the following form,

$$
\rho(\mathbf{r}, t)=<\Psi_{i o n}(t)\left|\sum_{j} \delta\left(\mathbf{r}-\mathbf{r}_{j}\right)\right| \Psi_{i o n}(t)>,
$$

where $\mathbf{r}_{j}$ denotes coordinate of $j$-th ion. The Fourier picture of the above density has the form,

$$
\tilde{\rho}(\mathbf{k}, t)=\int \rho(\mathbf{r}, t) e^{-i \mathbf{k} \cdot \mathbf{r}} d^{3} r=<\Psi_{i o n}(t)|\hat{\rho}(\mathbf{k})| \Psi_{\text {ion }}(t)>,
$$

where the 'operator' $\hat{\rho}(\mathbf{k})=\sum_{j} e^{-i \mathbf{k} \cdot \mathbf{r}_{j}}$.

Using the above notation one can rewrite $\hat{H}_{\text {ion }}$ in the following form, in an analogy to metallic plasmon description [1,2]:

$$
\begin{aligned}
\hat{H}_{i o n}= & \sum_{j=1}^{N}\left[-\frac{\hbar^{2} \nabla_{j}^{2}}{2 m}\right]-\frac{q^{\prime 2}}{(2 \pi)^{3}} \int d^{3} k \tilde{n}(\mathbf{k}) \frac{2 \pi}{k^{2}}\left(\hat{\rho^{+}}(\mathbf{k})+\hat{\rho}(\mathbf{k})\right) \\
& +\frac{q^{\prime 2}}{(2 \pi)^{3}} \int d^{3} k \frac{2 \pi}{k^{2}}\left[\hat{\rho^{+}}(\mathbf{k}) \hat{\rho}(\mathbf{k})-N\right]
\end{aligned}
$$

where: $\tilde{n}(\mathbf{k})=\int d^{3} r n(\mathbf{r}) e^{-i \mathbf{k} \cdot \mathbf{r}}$ (we have taken into account that $\left.\left.\frac{4 \pi}{k^{2}}=\int d^{3} r \frac{1}{r} e^{-i \mathbf{k} \cdot \mathbf{r}}\right), q^{\prime 2}=\frac{q^{2}}{\varepsilon}\right)$.

Utilizing this form of the effective ion Hamiltonian one can write out the dynamic equation in Heisenberg representation for ion density fluctuations,

$$
\frac{d^{2} \hat{\rho}(\mathbf{k})}{d t^{2}}=\frac{1}{(i \hbar)^{2}}\left[\left[\hat{\rho}(\mathbf{k}), \hat{H}_{i o n}\right], \hat{H}_{i o n}\right]
$$

in the following form,

$$
\begin{aligned}
& \frac{d^{2} \hat{\rho}(\mathbf{k})}{d t^{2}}=-\sum_{j} e^{-i \mathbf{k} \cdot \mathbf{r}_{j}}\left\{-\frac{\hbar^{2}}{m^{2}}\left(\mathbf{k} \cdot \nabla_{j}\right)^{2}+\frac{\hbar^{2} k^{2}}{m^{2}} i \mathbf{k} \cdot \nabla_{j}+\frac{\hbar^{2} k^{4}}{4 m^{2}}\right\} \\
& -\frac{4 \pi q^{\prime 2}}{m(2 \pi)^{3}} \int d^{3} p \tilde{n}(\mathbf{p}) \frac{\mathbf{k} \cdot \mathbf{p}}{p^{2}} \hat{\rho}(\mathbf{k}-\mathbf{p})-\frac{4 \pi q^{\prime 2}}{m(2 \pi)^{3}} \int d^{3} p \hat{\rho}(\mathbf{k}-\mathbf{p}) \frac{\mathbf{k} \cdot \mathbf{p}}{p^{2}} \hat{\rho}(\mathbf{p}) .
\end{aligned}
$$

If one takes into account that $\hat{\rho}(\mathbf{k}-\mathbf{p}) \hat{\rho}(\mathbf{p})=\delta \hat{\rho}(\mathbf{k}-\mathbf{p}) \delta \hat{\rho}(\mathbf{p})+\tilde{n}(\mathbf{k}-\mathbf{p}) \delta \hat{\rho}(\mathbf{p})+\delta \hat{\rho}(\mathbf{k}-\mathbf{p}) \tilde{n}(\mathbf{p})+$ $\tilde{n}(\mathbf{k}-\mathbf{p}) \tilde{n}(\mathbf{p})$ and $\tilde{n}(\mathbf{p}) \hat{\rho}(\mathbf{k}-\mathbf{p})=\tilde{n}(\mathbf{p}) \delta \hat{\rho}(\mathbf{k}-\mathbf{p})+\tilde{n}(\mathbf{p}) \tilde{n}(\mathbf{k}-\mathbf{p})$, where $\delta \hat{\rho}(\mathbf{k})=\hat{\rho}(\mathbf{k})-\tilde{n}(\mathbf{k})$ describes the 'operator' of local ion density fluctuation above the uniform distribution, one can rewrite Equation (9) as follows,

$$
\begin{aligned}
& \frac{d^{2} \delta \hat{\rho}(\mathbf{k})}{d t^{2}}=-\sum_{j} e^{-i \mathbf{k} \cdot \mathbf{r}_{j}}\left\{-\frac{\hbar^{2}}{m^{2}}\left(\mathbf{k} \cdot \nabla_{j}\right)^{2}+\frac{\hbar^{2} k^{2}}{m^{2}} i \mathbf{k} \cdot \nabla_{j}+\frac{\hbar^{2} k^{4}}{4 m^{2}}\right\} \\
& -\frac{4 \pi q^{\prime 2}}{m(2 \pi)^{3}} \int d^{3} p \tilde{n}(\mathbf{k}-\mathbf{p}) \frac{\mathbf{k} \cdot \mathbf{p}}{p^{2}} \delta \hat{\rho}(\mathbf{p})-\frac{4 \pi q^{\prime 2}}{m(2 \pi)^{3}} \int d^{3} p \delta \hat{\rho}(\mathbf{k}-\mathbf{p}) \frac{\mathbf{k} \cdot \mathbf{p}}{p^{2}} \delta \hat{\rho}(\mathbf{p}) .
\end{aligned}
$$

Hence, for the ion density fluctuation, $\delta \tilde{\rho}(\mathbf{k}, t)=<\Psi_{i o n}|\delta \hat{\rho}(\mathbf{k}, t)| \Psi_{\text {ion }}>=\tilde{\rho}(\mathbf{k}, t)-\tilde{n}(\mathbf{k})$, one can find,

$$
\begin{aligned}
& \frac{\partial^{2} \delta \tilde{\rho}(\mathbf{k}, t)}{\partial t^{2}}=-<\Psi_{i o n}\left|\sum_{j} e^{-i \mathbf{k} \cdot \mathbf{r}_{j}}\left\{-\frac{\hbar^{2}}{m^{2}}\left(\mathbf{k} \cdot \nabla_{j}\right)^{2}+\frac{\hbar^{2} k^{2}}{m^{2}} i \mathbf{k} \cdot \nabla_{j}+\frac{\hbar^{2} k^{4}}{4 m^{2}}\right\}\right| \Psi_{i o n}> \\
& -\frac{4 \pi q^{\prime 2}}{m(2 \pi)^{3}} \int d^{3} p \tilde{n}(\mathbf{k}-\mathbf{p}) \frac{\mathbf{k} \cdot \mathbf{p}}{p^{2}} \delta \tilde{\rho}(\mathbf{p}, t)-\frac{4 \pi q^{\prime 2}}{m(2 \pi)^{3}} \int d^{3} p \frac{\mathbf{k} \cdot \mathbf{p}}{p^{2}}<\Psi_{i o n}|\delta \hat{\rho}(\mathbf{k}-\mathbf{p}) \delta \hat{\rho}(\mathbf{p})| \Psi_{i o n}>.
\end{aligned}
$$


For small $k$, in analogy to the semiclassical approximation for electrons [1,2], the contributions of the second and third components to the first term on the right-hand-side of Equation (11) can be neglected as small in comparison to the first component. Small and thus negligible is also the third term in the right-hand-side of Equation (11), as involving a product of two $\delta \tilde{\rho}$ (which we assumed small, $\delta \tilde{\rho} / n<<1$ ). This approach corresponds to the RPA formulated for bulk metal [2] (note that $\delta \hat{\rho}(0)=0$ and the coherent RPA contribution of interaction is comprised by the last but one term in Equation (11)).

Within the RPA, Equation (11) thus attains the following shape,

$$
\begin{aligned}
\frac{\partial^{2} \delta \tilde{\rho}(\mathbf{k}, t)}{\partial t^{2}}= & \frac{2 k^{2}}{3 m}<\Psi_{i o n}\left|\sum_{j} e^{-i \mathbf{k} \cdot \mathbf{r}_{j}} \frac{\hbar^{2} \nabla_{j}^{2}}{2 m}\right| \Psi_{\text {vion }}> \\
& -\frac{4 \pi q^{\prime 2}}{m(2 \pi)^{3}} \int d^{3} p \tilde{n}(\mathbf{k}-\mathbf{p}) \frac{\mathbf{k} \cdot \mathbf{p}}{p^{2}} \delta \tilde{\rho}(\mathbf{p}, t),
\end{aligned}
$$

and due to the spherical symmetry,

$$
<\Psi_{i o n}\left|\sum_{j} e^{-i \mathbf{k} \cdot \mathbf{r}_{j}} \frac{\hbar^{2}}{m^{2}}\left(\mathbf{k} \cdot \nabla_{j}\right)^{2}\right| \Psi_{i o n}>\simeq \frac{2 k^{2}}{3 m}<\Psi_{i o n}\left|\sum_{j} e^{-i \mathbf{k} \cdot \mathbf{r}_{j}} \frac{\hbar^{2} \nabla_{j}^{2}}{2 m}\right| \Psi_{i o n}>.
$$

One can rewrite Equation (12) in the position representation,

$$
\begin{aligned}
& \frac{\partial^{2} \delta \tilde{\rho}(\mathbf{r}, t)}{\partial t^{2}}=-\frac{2}{3 m} \nabla^{2}<\Psi_{i o n}\left|\sum_{j} \delta\left(\mathbf{r}-\mathbf{r}_{j}\right) \frac{\hbar^{2} \nabla_{j}^{2}}{2 m}\right| \Psi_{i o n}> \\
& +\frac{\omega_{p}^{2}}{4 \pi} \nabla\left\{\Theta(a-r) \nabla \int d^{3} r_{1} \frac{1}{\left|\mathbf{r}-\mathbf{r}_{1}\right|} \delta \tilde{\rho}\left(\mathbf{r}_{1}, t\right)\right\} .
\end{aligned}
$$

In the case of metals it was next used the Thomas-Fermi formula to assess the averaged kinetic energy [2]:

$$
\begin{aligned}
& <\Psi_{i o n}\left|-\sum_{j} \delta\left(\mathbf{r}-\mathbf{r}_{j}\right) \frac{\hbar^{2} \nabla_{j}^{2}}{2 m}\right| \Psi_{i o n}>\simeq \frac{3}{5}\left(3 \pi^{2}\right)^{2 / 3} \frac{\hbar^{2}}{2 m}(\rho(\mathbf{r}, t))^{5 / 3} \\
& =\frac{3}{5}\left(3 \pi^{2}\right)^{2 / 3} \frac{\hbar^{2}}{2 m} n^{5 / 3} \Theta(a-r)\left[1+\frac{5}{3} \frac{\delta \tilde{\rho}(\mathbf{r}, t)}{n}+\ldots\right] .
\end{aligned}
$$

The Thomas-Fermi formula is addressed, however, to fermionic and degenerate quantum systems, as electrons in metals. For ionic systems such estimation of kinetic energy is inappropriate, because the ion concentration is usually much lower than that of electrons in metals and the system is not degenerated even if ions are fermions. The Maxwell-Boltzmann distribution should be applied instead of the Fermi-Dirac or Bose-Einstein ones. Independently of fermionic of bosonic statistics of ions, the Maxwell-Boltzmann distribution allows for the estimation of the averaged kinetic energy of ions located inside the spherical electrolyte with the radius $a$, in the following form,

$$
<\Psi_{i o n}\left|-\sum_{j} \delta\left(\mathbf{r}-\mathbf{r}_{j}\right) \frac{\hbar^{2} \nabla_{j}^{2}}{2 m}\right| \Psi_{i o n}>\simeq(n+\delta \rho(\mathbf{r}, t)) \Theta(a-r) \frac{3 k_{B} T}{2},
$$

where $k_{B}$ is the Boltzmann constant and $T$ the temperature. For ions of 3D shape of or of a linear shape, the inclusion of the rotational degrees of freedom result in factor of $\frac{6 k_{B} T}{2}$ or of $\frac{5 k_{B} T}{2}$, respectively, instead of $\frac{3 k_{B} T}{2}$ for the point-like ion model.

Using the formula (15) and taking into account that $\nabla \Theta(a-r)=-\frac{\mathbf{r}}{r} \delta(a-r)$, one can rewrite Equation (13) in the following manner,

$$
\begin{aligned}
& \frac{\partial^{2} \delta \tilde{\rho}(\mathbf{r}, t)}{\partial t^{2}}=\left[\frac{k_{B} T}{m} \nabla^{2} \delta \tilde{\rho}(\mathbf{r}, t)-\omega_{p}^{2} \delta \tilde{\rho}(\mathbf{r}, t)\right] \Theta(a-r) \\
& -\frac{k_{B} T}{m} \nabla\left\{[n+\delta \tilde{\rho}(\mathbf{r}, t)] \frac{\mathbf{r}}{r} \delta(a-r)\right\} \\
& -\left[\frac{k T}{m} \frac{\mathbf{r}}{r} \nabla \delta \tilde{\rho}(\mathbf{r}, t)+\frac{\omega_{p}^{2}}{4 \pi} \frac{\mathbf{r}}{r} \nabla \int d^{3} r_{1} \frac{1}{\left|\mathbf{r}-\mathbf{r}_{1}\right|} \delta \tilde{\rho}\left(\mathbf{r}_{1}, t\right)\right] \delta(a-r) .
\end{aligned}
$$


In the above formula $\omega_{p}$ is the bulk ion-plasmon frequency, $\omega_{p}^{2}=\frac{4 \pi n q^{\prime 2}}{m}$. The solution of Equation (16) can be decomposed into two parts related to the distinct domains-inside the sphere and on its surface,

$$
\delta \tilde{\rho}(\mathbf{r}, t)=\left\{\begin{array}{l}
\delta \tilde{\rho}_{1}(\mathbf{r}, t), \text { for } r<a, \\
\delta \tilde{\rho}_{2}(\mathbf{r}, t), \text { for } r \geq a,(r \rightarrow a+),
\end{array}\right.
$$

corresponding to the volume and surface excitations, respectively. These two parts of ion local density fluctuations satisfy the equations (according to Equation (16)),

$$
\frac{\partial^{2} \delta \tilde{\rho}_{1}(\mathbf{r}, t)}{\partial t^{2}}=\frac{k_{B} T}{m} \nabla^{2} \delta \tilde{\rho}_{1}(\mathbf{r}, t)-\omega_{p}^{2} \delta \tilde{\rho}_{1}(\mathbf{r}, t)
$$

and (here $\epsilon=0+$ )

$$
\begin{aligned}
& \frac{\partial^{2} \delta \tilde{\rho}_{2}(\mathbf{r}, t)}{\partial t^{2}}=-\frac{k_{B} T}{m} \nabla\left\{\left[n+\delta \tilde{\rho}_{2}(\mathbf{r}, t)\right] \frac{\mathbf{r}}{r} \delta(a+\epsilon-r)\right\} \\
& -\left[\frac{k_{B} T}{m} \frac{\mathbf{r}}{r} \nabla \delta \tilde{\rho}_{2}(\mathbf{r}, t)+\frac{\omega_{p}^{2}}{4 \pi} \frac{\mathbf{r}}{r} \nabla \int d^{3} r_{1} \frac{1}{\left|\mathbf{r}-\mathbf{r}_{1}\right|}\left(\delta \tilde{\rho}_{1}\left(\mathbf{r}_{1}, t\right) \Theta\left(a-r_{1}\right)\right.\right. \\
& \left.\left.+\delta \tilde{\rho}_{2}\left(\mathbf{r}_{1}, t\right) \Theta\left(r_{1}-a\right)\right)\right] \delta(a+\epsilon-r) .
\end{aligned}
$$

The Dirac delta in Equation (19) results due to the derivative of the Heaviside step function-the fictitious jellium distribution shape. In Equation (19) an infinitesimal shift, $\epsilon=0+$, is introduced to fulfil requirements of the Dirac delta definition (its singular point has to be an inner point of an open subset of the domain). This shift is only of a formal character and does not reflect any asymmetry. Some kind of asymmetry is, however, caused by the last term in Equation (16), the gradient-type term, which describes the electric field induced by ion density fluctuations. The electric field due to surface charges is zero inside the sphere, and therefore cannot influence the volume excitations. Conversely, the volume charge fluctuation-induced-electric-field can excite the surface fluctuations. This asymmetry is visible by comparison of Equations (18) and (19), for the volume and surface of plasmons, respectively. The equation for the volume of plasmons is completely independent of the surface of plasmons, whereas the volume plasmons contribute the equation for the surface plasmons.

The problem of separation between surface and volume plasmons has been thoroughly analyzed for metal clusters. For very small clusters the significance in this size-scale effect of the spill-out of electrons beyond the jellium edge makes the surface fuzzy resulting in coupling of volume and surface plasmon oscillations. By direct numerical simulations (TDLDA, i.e., the time dependent local density approximation) $[3,4]$ it has been proved that the volume-surface excitation mixing gradually disappears in larger clusters (with the number of electrons exceeding ca. 60) [3,4], which supports accuracy of semiclassical RPA description, within which volume plasmons can be separated from the surface ones (even though the latter can be excited by the former ones, due to the last term in Equation (19)). The volume-surface coupling, important for ultra-small radii of metallic clusters, when the shell effects and quantum spill-out are significant, completely disappear, however, with growing sphere dimension to the range of several nanometers in the case metals. We consider here the binary electrolyte system with radius of order of micrometer, when quantum effects specific for ultra-small clusters are negligible. Such an opportunity allows us to formulate an analytical RPA semiclassical description in the form of an oscillator equation, allowing for phenomenological inclusion of damping effects. The energy dissipation effects, though going beyond the RPA model, turned out to be the overwhelming physical property in the case of larger metallic nanospheres [1,5] (with $a>10 \mathrm{~nm}$ for $\mathrm{Au}$ or Ag) and also for much larger ionic systems, as we will demonstrate it below. 


\section{Solution of RPA Plasmon Equation: Volume and Surface Plasmon Frequencies in a Spherical Finite Ion System}

Equations (18) and (19) are solved for metallic nanospheres [1] and these solutions can be directly applied to ionic systems. To summarize briefly this analysis, both parts of the plasma fluctuation can represented as follows,

$$
\begin{aligned}
& \delta \tilde{\rho}_{1}(\mathbf{r}, t)=n\left[f_{1}(r)+F(\mathbf{r}, t)\right], \text { for } r<a, \\
& \delta \tilde{\rho}_{2}(\mathbf{r}, t)=n f_{2}(r)+\sigma(\Omega, t) \delta(r+\epsilon-a), \epsilon=0+, \text { for } r \geq a,(r \rightarrow a+),
\end{aligned}
$$

with initial conditions, $\left.F(\mathbf{r}, t)\right|_{t=0}=0,\left.\sigma(\Omega, t)\right|_{t=0}=0,\left(\Omega\right.$ is the spherical angle), $\left.\left(1+f_{1}(r)\right)\right|_{r=a}=$ $\left.f_{2}(r)\right|_{r=a}$ (continuity condition), $\left.F(\mathbf{r}, t)\right|_{r=a}=0, \int \rho(\mathbf{r}, t) d^{3} r=N$ (neutrality condition).

Neglecting the static contributions $f_{1,2}$ describing the spill-out effect, which sharply lowers with the system size growth [1], one can write out the time-dependent parts of the ion concentration fluctuations in the form,

$$
F(\mathbf{r}, t)=\sum_{l=1}^{\infty} \sum_{m=-l}^{l} \sum_{i=1}^{\infty} A_{l m n} j_{l}\left(k_{n l} r\right) Y_{l m}(\Omega) \sin \left(\omega_{l i} t\right),
$$

and

$$
\begin{aligned}
& \sigma(\Omega, t)=\sum_{l=1}^{\infty} \sum_{m=-l}^{l} \frac{B_{l m}}{a^{2}} Y_{l m}(\Omega) \sin \left(\omega_{0 l} t\right) \\
& +\sum_{l=1}^{\infty} \sum_{m=-l}^{l} \sum_{i=1}^{\infty} A_{l m n} \frac{(l+1) \omega_{p}^{2}}{l \omega_{p}^{2}-(2 l+1) \omega_{l i}^{2}} Y_{l m}(\Omega) n_{e} \int_{0}^{a} d r_{1} \frac{r_{l}^{l+2}}{a^{l+2}} j_{l}\left(k_{l i} r_{1}\right) \sin \left(\omega_{l i} t\right),
\end{aligned}
$$

where $j_{l}(\xi)=\sqrt{\frac{\pi}{2 \xi}} I_{l+1 / 2}(\xi)$ is the spherical Bessel function, $Y_{l m}(\Omega)$ is the spherical function, $\omega_{l i}=$ $\omega_{p} \sqrt{1+\frac{k_{B} T x_{l i}^{2}}{\omega_{p}^{2} a^{2} m}}$ are the frequencies of ion volume self-oscillations (volume plasmon frequencies), $x_{l i}$ are the nodes of the Bessel function $j_{l}(\xi)$ numerated with $i=1,2,3 \ldots$ (cf. Figure 2), $k_{l i}=x_{l i} / a$, $\omega_{l 0}=\omega_{p} \sqrt{\frac{l}{2 l+1}}$ are the frequencies of ion surface self-oscillations (surface plasmon frequencies). The derivation of the self-frequencies for ionic plasmon oscillations is presented with all details in Appendix A. Amplitudes $A_{l m i}$ and $B_{l m}$ are arbitrary in the homogeneous problem and can be adjusted to the initial conditions.
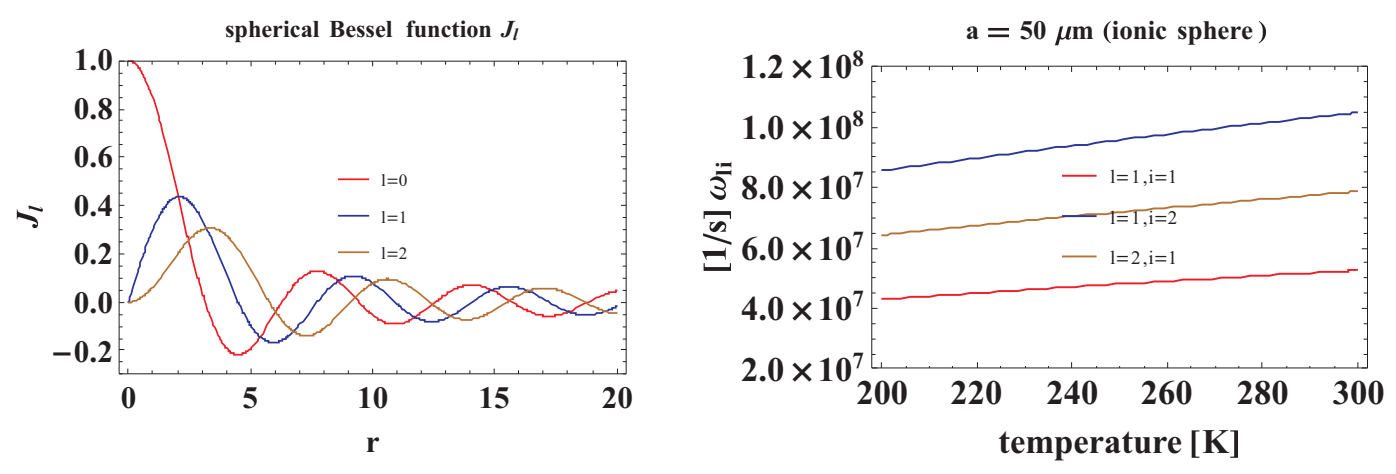

Figure 2. The spherical Bessel functions $J_{l}(r)$ for $l=0,1,2$ displaying possible charge density fluctuations in the sphere along the sphere radius $r$ (arbitrary units) for volume plasmon modes; the angular distribution for these modes is governed by the real spherical functions $Y_{l m}(\Omega)$ similarly as for the surface plasmon modes (cf. Figure 1 right). The exemplary temperature dependence of self-frequencies of volume plasmon modes $\omega_{l i}, l i=11,12,21$, for diluted electrolyte $n \simeq 10^{14} 1 / \mathrm{m}^{3}$ and ion mass $\sim 10^{4} m_{e}, a \sim 50 \mu \mathrm{m}$-right. 
The function $F(\mathbf{r}, t)$ describes volume plasmon oscillations, whereas $\sigma(\Omega, t)$ describes the surface plasmon oscillations. Let us emphasize that the first term in the Equation (22) corresponds to surface self-oscillations, while the second term describes the surface oscillations induced by the volume plasmons. The frequencies of the surface self-oscillations equal to,

$$
\omega_{0 l}=\omega_{p} \sqrt{\frac{l}{2 l+1}}
$$

which, for $l=1$, is the dipole type surface oscillation frequency, described for metallic nanosphere by Mie [6], $\omega_{01}=\omega_{p} / \sqrt{3}$.

Ionic Surface Ion Plasmon Frequencies for the Electrolyte Nanosphere Embedded in a Dielectric Medium, with $\varepsilon_{1}>1$

One can now include the influence of a dielectric surroundings (in general, distinct from the inner one of considered ionic system) on plasmons in this system. In order to do it, let us assume that ions on the surface $(r=a+$, i.e., $r \geq a, r \rightarrow a)$ interact with Coulomb forces renormalized by the relative dielectric permittivity $\varepsilon_{1}>1$ (distinct from $\varepsilon$ for inner medium). Thus a small modification of Equation (19) is of order,

$$
\begin{aligned}
& \frac{\partial^{2} \delta \tilde{\rho}_{2}(\mathbf{r})}{\partial t^{2}}=-\frac{2}{3 m} \nabla\left\{\left[\frac{3}{5} \epsilon_{F} n+\epsilon_{F} \delta \tilde{\rho}_{2}(\mathbf{r}, t)\right] \frac{\mathbf{r}}{r} \delta(a+\epsilon-r)\right\} \\
& -\left[\frac{2}{3} \frac{\epsilon_{F}}{m} \frac{\mathbf{r}}{r} \nabla \delta \tilde{\rho}_{2}(\mathbf{r}, t)+\frac{\omega_{p}^{2}}{4 \pi} \frac{\mathbf{r}}{r} \nabla \int d^{3} r_{1} \frac{1}{\left|\mathbf{r}-\mathbf{r}_{1}\right|}\left(\delta \tilde{\rho}_{1}\left(\mathbf{r}_{1}, t\right) \Theta\left(a-r_{1}\right)\right.\right. \\
& \left.\left.+\frac{1}{\varepsilon_{1}} \delta \tilde{\rho}_{2}\left(\mathbf{r}_{1}, t\right) \Theta\left(r_{1}-a\right)\right)\right] \delta(a+\epsilon-r),
\end{aligned}
$$

Note that Equation (18) is not affected by the outer medium. The solution of the above equation is of the same form as that for Equation (19), but with the renormalized surface plasmon frequencies,

$$
\omega_{0 l}=\omega_{p} \sqrt{\frac{l}{2 l+1} \frac{1}{\varepsilon_{1}}} .
$$

\section{Damping of Plasmon Oscillations in Ionic Systems}

The semiclassical RPA treatment of plasmon excitations in finite ion systems as presented above, does not account for plasmon damping. The damping of plasmon oscillations can be, however, included in a phenomenological manner, by addition of an attenuation term to plasmon dynamic equations, i.e., the term, $-\frac{2}{\tau_{0}} \frac{\partial \delta \rho(\mathbf{r}, t)}{\partial t}$, added to the r.h.s. of both Equations (18) and (19), taking advantage of their oscillatory form. The damping rate $\frac{1}{\tau_{0}}$ accounts for ion scattering losses and can be approximated in analogy to metallic systems, by inclusion of energy dissipation caused by irreversible its transformation into heat via various microscopic channels similar to those for Ohmic resistivity [7],

$$
\frac{1}{\tau_{0}} \simeq \frac{v}{2 \lambda_{b}}+\frac{C v}{2 a}
$$

where $a$ is the sphere radius, $v$ is the mean velocity of ions, $v=\sqrt{\frac{4 k_{B} T}{3 m}}, \lambda_{b}$ is the ion mean free path in bulk electrolyte material the same as the sphere is made of (including scattering of ions on other ions, and on solvent particles and admixtures). The second term in Equation (26) accounts for the scattering of ions on the boundary of the finite ionic system, the sphere with the radius $a$, and the constant $C$ is of order of unity [7].

In order to explicitly express a forcing field which moves ions in the system, the inhomogeneous time dependent term should be added to homogeneous Equations (18) and (19). The forcing field may be the time dependent electric field. If one considers it as the electric component of the incident $\mathrm{EM}$ wave then the comparison of the resonant wavelength with the system size is of order. Similarly 
as for metallic nanospheres also for finite ionic systems the surface plasmon resonant wavelength highly exceeds the system dimension and the forcing field is practically uniform along the whole system. Such a perturbation can excite only surface dipole plasmons, i.e., the mode with $l=1$, which can be described by the function $Q_{1 m}(t)(l=1$ and $m$ are angular momentum numbers related to the assumed spherical symmetry of the system). The corresponding dynamical equation for surface plasmons reduced to only mode $Q_{1 m}(t)$ has the following form,

$$
\begin{aligned}
& \frac{\partial^{2} Q_{1 m}(t)}{\partial t^{2}}+\frac{2}{\tau_{0}} \frac{\partial Q_{1 m}(t)}{\partial t}+\omega_{1}^{2} Q_{1 m}(t) \\
& =\sqrt{\frac{4 \pi}{3}} \frac{q n}{m}\left[E_{z}(t) \delta_{m, 0}+\sqrt{2}\left(E_{x}(t) \delta_{m, 1}+E_{y}(t) \delta_{m,-1}\right)\right],
\end{aligned}
$$

where $\omega_{1}=\frac{\omega_{p}}{\sqrt{3 \varepsilon_{1}}}$ (it is a dipole surface plasmon frequency, i.e., the Mie frequency [6], $\varepsilon_{1}$ is the dielectric susceptibility of the system surroundings). Because only $Q_{1 m}$ contribute to the plasmon response to the homogeneous electric field, thus the effective ion density fluctuation has the form [1],

$$
\delta \rho(\mathbf{r}, t)=\left\{\begin{array}{l}
0, r<a, \\
\sum_{m=-1}^{1} Q_{1 m}(t) Y_{1 m}(\Omega) r \geq a, r \rightarrow a+,
\end{array}\right.
$$

where $Y_{l m}(\Omega)$ is the spherical function with $l=1$. One can also explicitly calculate the dipole $\mathbf{D}(t)$ corresponding to surface plasmon oscillations given by Equation (28),

$$
\left\{\begin{array}{l}
D_{x}(t)=q \int d^{3} r x \delta \rho(\mathbf{r}, t)=\frac{\sqrt{2 \pi}}{\sqrt{3}} e Q_{1,1}(t) a^{3}, \\
D_{y}(t)=q \int d^{3} r y \delta \rho(\mathbf{r}, t)=\frac{\sqrt{2 \pi}}{\sqrt{3}} e Q_{1,-1}(t) a^{3}, \\
D_{z}(t)=q \int d^{3} r z \delta \rho(\mathbf{r}, t)=\frac{\sqrt{4 \pi}}{\sqrt{3}} e Q_{1,0}(t) a^{3} .
\end{array}\right.
$$

The dipole $\mathbf{D}(t)$ satisfies the equation (if one rewrites Equation (27)),

$$
\left[\frac{\partial^{2}}{\partial t^{2}}+\frac{2}{\tau_{0}} \frac{\partial}{\partial t}+\omega_{1}^{2}\right] \mathbf{D}(t)=\frac{a^{3} 4 \pi q^{2} n}{3 m} \mathbf{E}(t)=\varepsilon a^{3} \omega_{1}^{2} \mathbf{E}(t)
$$

One can notice that the dipole (29) scales as the system volume, $\sim a^{3}$, which may be interpreted that all ions actually contribute to the surface plasmon oscillations. This is connected with the fact that the surface modes correspond to uniform translation-type oscillations of ions in the system, when inside the sphere the charge of ions is exactly compensated by oppositely signed ions, whereas the not balanced charge density occurs only on the surface despite that all ions oscillate. For the volume plasmons the non-compensated charge density fluctuation are present also inside the sphere as volume plasmon modes have the compressional character with not balanced charge fluctuations along the sphere radius.

The scattering effects accounted for by the approximate formula (26) cause damping of plasmons especially strong for small size of the system due to the nanosphere-edge scattering contribution proportional to $\frac{1}{a}$. This term is, however, of lowering significance with the radius growth. We will show that radiation losses resulted due to accelerated movement of ions scales as $a^{3}$, and for rising $a$ these irradiative energy losses quickly dominate plasmon attenuation. Due to opposite size dependence of scattering and irradiation contributions to the plasmon damping one can thus observe the cross-over in damping with respect to its size dependence, as it is depicted in Figure 3. One can also determine the radius $a^{*}$ for which the total attenuation rate for surface plasmons is minimal, $a^{*}=\left(\frac{3^{3 / 2} C c^{3} v}{2 \omega_{1} \omega_{p}^{3}}\right)^{1 / 4}$. The system size $a^{*}$ for two distinct ionic systems are listed in Table 1. 

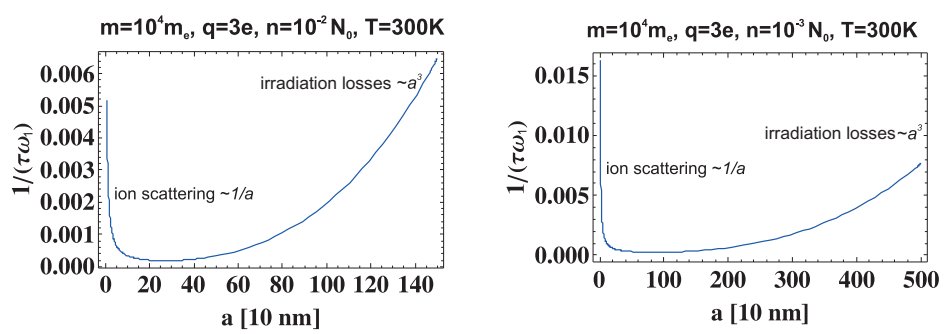

Figure 3. The cross-over in ionic system-size dependence of damping rate for surface plasmons, for $T=300 \mathrm{~K}, m=10^{4} m_{e}, q=3 e, n=10^{-2} N_{0}\left(N_{0}\right.$ is the concentration of one molar electrolyte) (left) and for $n=10^{-3} N_{0}$ (right); ion scattering losses, $\sim \frac{1}{a}$, are of lowering importance with growth of the electrolyte sphere size ( $a$ is the sphere radius), whereas the irradiation losses dominate at larger size, as it initially grows as $a^{3}$; in the size-region close to the cross-over the perturbative treatment for Lorentz friction perfectly coincides with the exact approach.

Table 1. The ion-system parameters assumed for calculation of damping rates and self-frequency for dipole surface plasmons.

\begin{tabular}{llll}
\hline Material & Ionic System & Sample 1 & Sample 2 \\
\hline ion concentration & $n\left(N_{0}\right.$ is one molar electr.) & $10^{-2} N_{0}$ & $10^{-3} N_{0}$ \\
effective ion mass & $m\left(m_{e}\right.$ electron mass) & $10^{4} m_{e}$ & $10^{4} m_{e}$ \\
charge of effective ion & $q / \sqrt{\varepsilon}$ & $3 e$ & $3 e$ \\
temperature & $T$ & $300 \mathrm{~K}$ & $300 \mathrm{~K}$ \\
mean velocity of ions & $v=\sqrt{\frac{3 k T}{m}}$ & $1168 \mathrm{~m} / \mathrm{s}$ & $1168 \mathrm{~m} / \mathrm{s}$ \\
bulk plasmon frequency & $\omega_{p}$ & $9.3 \times 10^{13} 1 / \mathrm{s}$ & $2.93 \times 10^{12} 1 / \mathrm{s}$ \\
dielectric constant of souroundings & $\varepsilon_{1}$ & 2 & 2 \\
Mie frequency & $\omega_{1}=\omega_{p} / \sqrt{3 \varepsilon_{1}}$ & $3.8 \times 10^{13} 1 / \mathrm{s}$ & $1.2 \times 10^{12} 1 / \mathrm{s}$ \\
constant in Equation (26) & $C$ & 2 & 2 \\
bulk mean free path (room temp.) & $\lambda_{b}$ & $0.5 \mu \mathrm{m}$ & $0.1 \mu \mathrm{m}$ \\
radius for minimal damping & $a^{*}=\left(\frac{3^{3 / 2} C c^{3} v}{2 \omega_{1} \omega_{p}^{3}}\right)$ & $2.7 \times 10^{-7} \mathrm{~m}$ & $8.6 \times 10^{-7} \mathrm{~m}$ \\
\hline
\end{tabular}

The irradiation of energy of the oscillating dipole is expressed by the so-called Lorentz friction [8], i.e., the effective electric field slowing down the motion of charges,

$$
\mathbf{E}_{L}=\frac{2 \sqrt{\varepsilon}}{3 c^{3}} \frac{\partial^{3} \mathbf{D}(t)}{\partial t^{3}} .
$$

Hence, we can rewrite Equation (30) including the Lorentz friction term,

$$
\left[\frac{\partial^{2}}{\partial t^{2}}+\frac{2}{\tau_{0}} \frac{\partial}{\partial t}+\omega_{1}^{2}\right] \mathbf{D}(t)=\varepsilon a^{3} \omega_{1}^{2} \mathbf{E}(t)+\varepsilon a^{3} \omega_{1}^{2} \mathbf{E}_{L}
$$

or for $\mathbf{E}=0$,

$$
\left[\frac{\partial^{2}}{\partial t^{2}}+\omega_{1}^{2}\right] \mathbf{D}(t)=\frac{\partial}{\partial t}\left[-\frac{2}{\tau_{0}} \mathbf{D}(t)+\frac{2}{3 \omega_{1}}\left(\frac{\omega_{p} a}{c \sqrt{3}}\right)^{3} \frac{\partial^{2}}{\partial t^{2}} \mathbf{D}(t)\right]
$$

Now one can apply the perturbation method for solution of Equation (33) when the r.h.s. of this equation is treated as a perturbation. In zeroth step of the perturbation we have $\left[\frac{\partial^{2}}{\partial t^{2}}+\omega_{1}^{2}\right] \mathbf{D}(t)=0$, from which $\frac{\partial^{2}}{\partial t^{2}} \mathbf{D}(t)=-\omega_{1}^{2} \mathbf{D}(t)$. Hence, for the first step of the perturbation, we put the latter formula to the r.h.s. of Equation (33), i.e.,

$$
\left[\frac{\partial^{2}}{\partial t^{2}}+\frac{2}{\tau} \frac{\partial}{\partial t}+\omega_{1}^{2}\right] \mathbf{D}(t)=0
$$


where

$$
\frac{1}{\tau}=\frac{1}{\tau_{0}}+\frac{\omega_{1}}{3}\left(\frac{\omega_{p} a}{c \sqrt{3}}\right)^{3} .
$$

Within the first step of perturbation, the Lorentz friction can be included into the total attenuation rate $\frac{1}{\tau}$. Nevertheless, this approximation is justified only for sufficiently small perturbations, i.e., when the second term in Equation (35), proportional to $a^{3}$, is small enough to fulfill the perturbation restrictions. The related limiting value, $\tilde{a}$, of the ionic system size depends on the ion concentration, charge, mass, dielectric susceptibility, as is exemplified below in the following subsection.

The solution of Equation (34) is of the form $\mathbf{D}(t)=\mathbf{A} e^{-t / \tau} \cos \left(\omega_{1}^{\prime} t+\phi\right)$, where $\omega_{1}^{\prime}=$ $\omega_{1}^{\prime} \sqrt{1-\frac{1}{\left(\omega_{1} \tau\right)^{2}}}$, which gives the red shift of the plasmon resonance due to strong, $\sim a^{3}$, growth of the attenuation caused by the irradiation. The Lorentz friction term in Equation (35) dominates plasmon damping for $a \leq \tilde{a}$ due to this $a^{3}$ dependence-cf. Figure 3. The plasmon damping grows rapidly with $a$ and this results in the pronounced red-shift of the resonance frequency.

Exact Inclusion of the Lorentz Damping to the Attenuation of Ionic Dipole Surface Plasmons

Now we will consider the dynamic equation for surface plasmons in the ionic spherical system (33) with the Lorentz friction term, but without application of the perturbation method. We change to dimensionless variable $t \rightarrow t^{\prime}=\omega_{1} t$. Then Equation (33) attains the form,

$$
\frac{\partial^{2} \mathbf{D}\left(t^{\prime}\right)}{\partial t^{\prime 2}}+\frac{2}{\tau_{0} \omega_{1}} \frac{\partial \mathbf{D}\left(t^{\prime}\right)}{\partial t^{\prime}}+\mathbf{D}\left(t^{\prime}\right)=\frac{2}{3}\left(\frac{\omega_{p} a}{v \sqrt{3}}\right)^{3} \frac{\partial^{3} \mathbf{D}\left(t^{\prime}\right)}{\partial t^{\prime 3}} .
$$

In the case of the solution of Equation (36) by the perturbation method we get the renormalized attenuation rate for the effective damping term, $\frac{1}{\omega_{1} \tau_{0}}+\frac{1}{3}\left(\frac{\omega_{p} a}{v \sqrt{3}}\right)^{3}$. This term quickly achieves the value 1, for which the oscillator falls down into the over-damped regime. For system parameters as assumed for Figure 3, the achievement by the attenuation rate of the value equaled to 1 takes place at $25.5 \mu \mathrm{m}$ and $8 \mu \mathrm{m}$ for $n=10^{-3} N_{0}$ and $n=10^{-2} N_{0}$, respectively. At these values the frequency $\omega_{1}^{\prime}=\omega_{1}^{\prime} \sqrt{1-\frac{1}{\left(\omega_{1} \tau\right)^{2}}}$ goes to zero, which indicates an apparent artifact of the perturbation method. To verify how behaves the exact damped frequency in the considered system one has to solve the dynamical equation without any approximations. As this equation is of the third order linear differential equation, one can find its solution in the form, $\sim e^{i \Omega t^{\prime}}$, with the analytical expressions for three possible values of the exponent,

$$
\begin{aligned}
\Omega_{1}= & -\frac{i}{3 g}-\frac{i 2^{1 / 3}(1+6 g u)}{3 g\left(2+27 g^{2}+18 g u+\sqrt{4(-1-6 g u)^{3}+\left(2+27 g^{2}+18 g u\right)^{2}}\right)^{1 / 3}} \\
& -\frac{i\left(2+27 g^{2}+18 g u+\sqrt{4(-1-6 g u)^{3}+\left(2+27 g^{2}+18 g u\right)^{2}}\right)^{1 / 3}}{3 \times 2^{1 / 3} g} \in \operatorname{im}(=i \alpha), \\
\Omega_{2}= & -\frac{i}{3 g}+\frac{i(1+i \sqrt{3})(1+6 g u)}{3 \times 2^{2 / 3} g\left(2+27 g^{2}+18 g u+\sqrt{4(-1-6 g u)^{3}+\left(2+27 g^{2}+18 g u\right)^{2}}\right)^{1 / 3}} \\
& +\frac{i(1-i \sqrt{3})\left(2+27 g^{2}+18 g u+\sqrt{4(-1-6 g u)^{3}+\left(2+27 g^{2}+18 g u\right)^{2}}\right)^{1 / 3}}{6 \times 2^{1 / 3} g}=\omega+i \frac{1}{\tau}, \\
\Omega_{3}= & \left.-\frac{i}{3 g}+\frac{i(1-i \sqrt{3})(1+6 g u)}{3 \times 2^{2 / 3} g\left(2+27 g^{2}+18 g u+\sqrt{4(-1-6 g u)^{3}+\left(2+27 g^{2}+18 g u\right)^{2}}\right.}\right)^{1 / 3} \\
& +\frac{i(1+i \sqrt{3})\left(2+27 g^{2}+18 g u+\sqrt{4(-1-6 g u)^{3}+\left(2+27 g^{2}+18 g u\right)^{2}}\right)^{1 / 3}}{6 \times 2^{1 / 3} g}=-i \frac{1}{\tau},
\end{aligned}
$$

where $u=\frac{1}{\tau_{0} \omega_{1}}$ and $g=2 / 3\left(\frac{a \omega_{p}}{c \sqrt{3 \varepsilon_{1}}}\right)^{3}$.

In Figure 4 we have plotted the damping rate $(\operatorname{Im} \Omega)$ and the self-frequency $(\operatorname{Re} \Omega)$ (also translated for resonance wavelength-right panels) with respect to the system radius $a$. For comparison, the approximate perturbative solutions are plotted-the blue line, whereas the exact solution of 
Equation (36) is plotted in the red line. The blue line finishes at $a_{\text {limit }}$, when the attenuation rate within the perturbation approach reaches the critical value 1 (then $\lambda \rightarrow \infty$ ). For the accurate solution of Equation (36) this singular behavior completely disappears and the oscillating solution, $e^{i \Omega t}$, exists for larger $a$ as well.
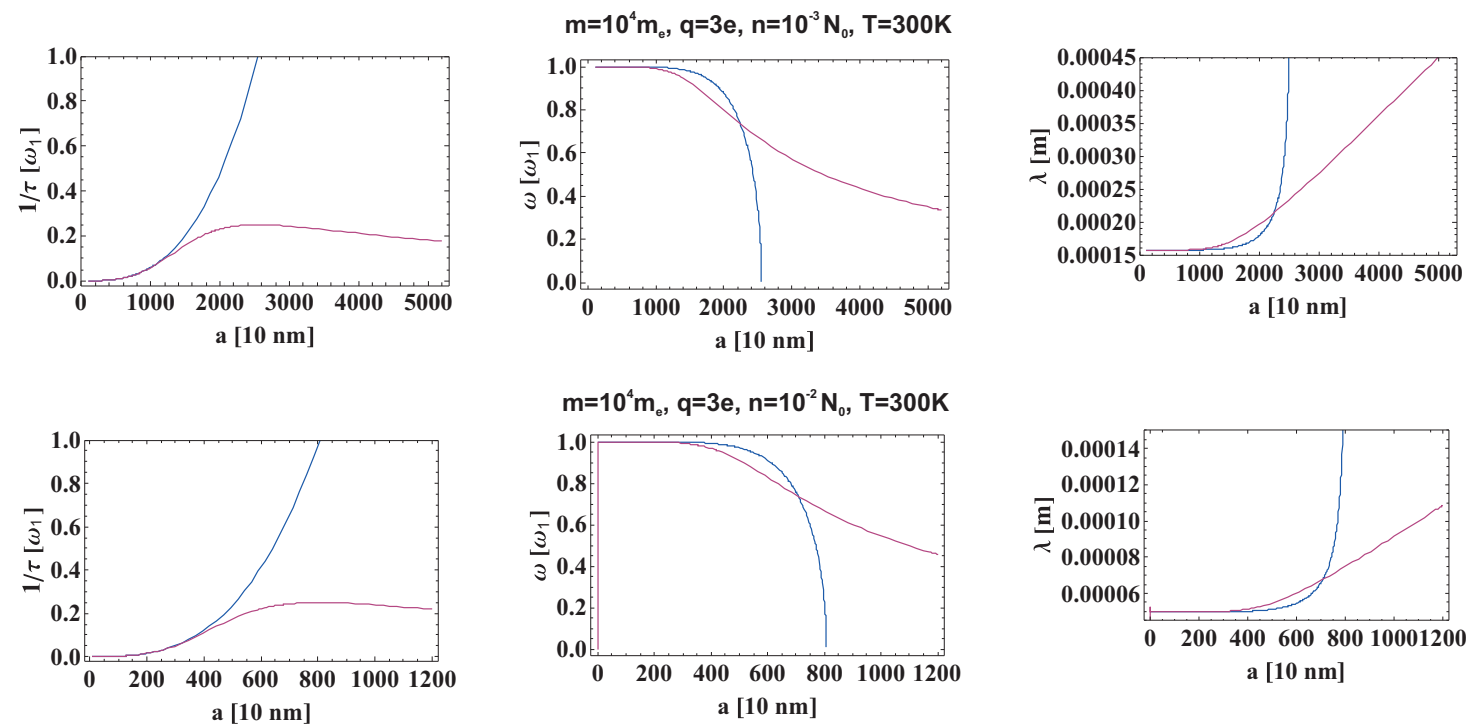

Figure 4. Comparison of the damping rate and the resonance frequency (transformed also in the resonance photon wavelength—in right panels), i.e., the damping rate and frequency (wavelength) of oscillating solution of Equation (36), exact (red line) and approximate upon perturbation approach (blue line), both with respect to the ionic finite system radius $a$.

We notice that the red-shift of the plasmon resonance is strongly overestimated in the framework of the perturbative approach to the Lorentz friction unless $a<\tilde{a}$, where $\tilde{a}$ is sensitive to ionic system parameters and especially to ion concentration (as is demonstrated in Figure 4).

Let us emphasize that the equation (36) has in general two types of particular solutions, $e^{i \Omega t^{\prime}}$, with complex self-frequencies $\Omega$. The solutions given by $\Omega_{2}$ and $\Omega_{3}$ are of oscillating type with damping ( $i \Omega_{2}$ and $i \Omega_{3}$ are mutually conjugated, thus $\Omega_{2}$ and $\Omega_{3}$ have the real parts of opposite sign, whereas the same imaginary parts, the latter is positive displaying the damping rate) and the second one-given by $\Omega_{1}$, which turns out to be an unstable exponentially rising solution (negative imaginary solution). This unstable solution is the well-known artifact in the Maxwell electrodynamics (cf. e.g., chapter 75 in [8]) and corresponds to the infinite self-acceleration of the free charge due to Lorentz friction force. More specifically, this artificial instability illustrates the singular solution of the equation $m \dot{\mathbf{v}}=$ const. $\times \ddot{\mathbf{v}}$, which is associated with a formal renormalization of the field-mass of the charge-infinite for point-like charge and canceled in an artificial manner by arbitrary assumed negative infinite non-field mass, resulting in ordinary mass of e.g., an electron, which is, however, not defined mathematically in a proper way. This unphysical singular particular solution should be thus discarded. The other oscillatory type solution resembles the solution of the ordinary damped harmonic oscillator, though with distinct attenuation rate and frequency. They are expressed by analytical formulae for $\Omega_{2}$ and $\Omega_{3}$ by Equations (37) and then are calculated for various $a$ and compared with the corresponding quantities found within the perturbation approach. This comparison is presented in Figure 4. From this comparison it is clearly visible that application of the perturbation approach leads to high overestimation of the damping rate for $a>\tilde{a}$. Therefore, we can conclude that the usage of the approximate formula for the Lorentz friction damping in the form (35) is justified up to $a \simeq \tilde{a}$, while for $a>\tilde{a}$, these approximate values strongly differ from the exact ones. The value $\tilde{a}<a_{\text {limit }}$ sharply depends on ionic system parameters and approximately $\tilde{a} \simeq \frac{a_{\text {limit }}}{2}$. 


\section{Plasmon-Polariton in the Chain of Finite Ionic Systems-Model of the Saltatory Conduction in Myelinated Axons}

The effective and quick transduction of the action potential through long myelinated axons in neurons of the peripheral nervous system and in the white matter of the central nervous system undeniably stands behind the motorics and other communication functions of the body. The acceleration of the signal transduction is associated here with jumping of it between consecutive so-called Ranvier nodes separated by myelinated sectors of the length of ca. $100 \mu \mathrm{m}$. The nature of this signal jumping, called as the saltatory conduction, is not explained as of yet as its timing exceeds conventional models of electrical signaling in neurons. Despite the very deep current recognition in mechanisms of the nerve functioning, including the action potential spike formation mechanism on the Ranvier node [9] and the model of the diffusion-like conduction of ion current in dendrites and non-myelinated axons according to the so-called cable theory originated by W. Thompson (1854) [10], cf. Figure 5 (with various modifications [11,12]), the saltatory conduction observed in myelinated axons [13] is still unclear as being apparently beyond the cable theory explanation ability and other possible mechanism is searched $[9,14]$.

\section{scheme for the cable model of nerve signaling in nonmyelinated axons and in dendrites}

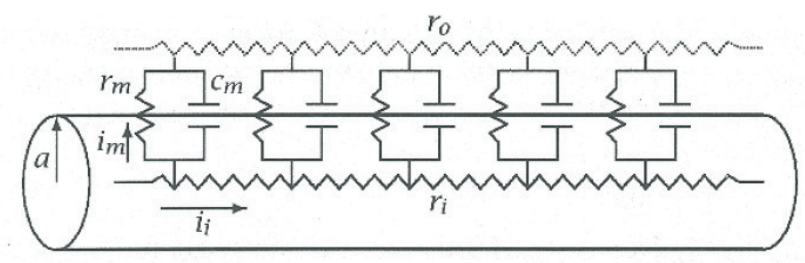

Figure 5. Schematic presentation of the cable model of nervous signal transduction in dendrites and in non-myelinated axons [9,12]; a capacity across the neuron membrane, $c_{m}$, together with across-membrane resistivity, $r_{m}$, create a series of coupled RC circuits; consecutive local charging and discharging of the capacitors $c_{m}$ results in diffusion type $[10,13]$ transduction of ion current $i_{i}$ inside the dendrite or non-myelinated axon; because of relatively large longitudinal resistivity $r_{i(o)}$ of inside (outside) cytosol as well as of the value $c_{m}$ and $r_{m}$ across the cell membrane the velocity of the diffusive current is limited to $1-3 \mathrm{~m} / \mathrm{s}[9,10,12]$.

In order to solve the problem of the saltatory conduction we develop a new and original model for this mysterious conduction basing on the kinetic properties of collective plasmon-polariton modes propagating along linear and periodically modified electrolyte system, which in the case of an axon is the thin cord of the nerve cell periodically wrapped by Schwann cells creating periodic and relatively thick myelin sheath (Schwann cells myelinate axons in the peripheral system, whereas in the central neural system the white substance is built from axons myelinated by the oligodendrocyte cells $[9,11,12])$. Plasmon-polaritons were investigated and understood based on the well-developed domain of plasmonics $[15,16]$, especially of nano-plasmonics applied to long range low-damped propagation of plasmon-polaritons along metallic nano-chains [17]. The main properties of these collective excitations, occurring on the conductor/insulator interface $[18,19]$ by hybridization of the surface plasmons (i.e., the electron density fluctuations on the metal surface) with the electromagnetic wave, are as follows: (1) much lower velocity of plasmon-polariton in comparison to the light velocity, which gives much shorter wavelength of plasmon-polariton than the light wavelength with the same frequency, (2) the related strong discrepancy of momenta of plasmon-polaritons and photons with the same energy causes that the external electromagnetic wave does not interact with plasmon-polariton, i.e., photons cannot be excited or absorbed by plasmon-polariton due to momentum conservation constraint, (3) all the electromagnetic field associated with propagation of plasmon-polariton is compressed to the tunnel-volume of the chain, especially to the space between chain elements, 
(4) all the radiative losses are quenched and plasmon-polariton attenuation is only due to Ohmic losses of oscillating charge carriers (electrons in metal), which makes metallic nano-chains almost perfect waveguides for plasmon-polaritons, (5) long range and practically undamped propagation of plasmon-polariton is observed experimentally in metallic nano-chains $[17,20]$. Plasmon-polaritons in metallic nanostructures are regarded as prospective for future applications in opto-electronics where conversion of the light signal onto plasmon-polariton signal allows for avoidance of the diffraction constraints strongly limiting miniaturization of conventional opto-electronic devices (as the nano scale of electron confinement inconveniently meets with several orders larger scale of the wavelength of light with energy similar to the electrons nano-confined) $[19,21]$.

Some of the unique properties of plasmon-polariton in metallic nano-chains are of high interest as they can be repeated in periodic linear alignments of electrolyte systems with ions instead of electrons as charge carriers. According to larger mass of ions in comparison to electrons and lower concentration of ions in electrolytes than of electrons in metals, the plasmon resonances in finite ionic systems (e.g., liquid electrolyte confined to the finite volume by appropriately formed membranes frequent in biological cell structures) occur in a different to metals scale of micrometers instead of nanometers and for several orders lower frequency (depending on the ion concentration).

For spherical electrolyte system one deals with the surface and volume plasmons in analogy to metallic nanosphere. The ionic surface plasmon frequencies are given for multipole $l$-th mode by the formula $\omega_{l}=\omega_{p} \sqrt{\frac{l}{\varepsilon(2 l+1)}}$ with the bulk plasmon frequency $\omega_{p}=\sqrt{\frac{4 \pi q^{2} n}{m}}$ ( $n$ is ion concentration, $q$ and $m$ are ion charge and mass, respectively, $\varepsilon$ is the dielectric relative permittivity of the surroundings). For dipole surface plasmons $(l=1)$, it resolves to the Mie-type formula, $[1,6] \omega_{1}=\frac{\omega_{p}}{\sqrt{3 \varepsilon}}$. These plasmon oscillations intensively irradiate their own energy and are quickly damped due to Lorentz friction losses (i.e., due to irradiation of EM wave by oscillating charges [8,22]) which for large systems with large number of ions participating in plasmon oscillations (thus strengthening the Lorentz friction) are much greater than the Joule-heat dissipation caused by Ohmic losses due to carriers scattering (scattering of ions on other ions, solvent and admixture atoms and on boundary of the system), as illustrated in Figure 3, where the crossover in the size dependence of plasmon damping rate is visible (similar as for metal nanospheres [23,24]).

Surprisingly, in the linear chain of spherical ionic systems the irradiation losses are completely reduced to zero, exactly in the same manner as in metallic chains [25-27]. The irradiation energy losses expressed by the Lorentz friction $[8,22]$ are ideally compensated by the income of the energy due to radiation of all other spheres in the chain. In the result, the radiative losses are ideally balanced and there remains only relatively small Ohmic irreversible energy dissipation due to ion scattering. The collective surface dipole plasmon-polariton can thus propagate in the chain with strongly reduced damping and if energy is permanently supplemented in a minor scale to balance only the Ohmic losses, this propagation could occur on arbitrarily long distances without any damping.

\section{Plasmon-Polariton Propagation in Linear Periodic Ionic System}

We propose [28] to apply the model of dipole plasmon-polariton excitations in the linear chain of electrolyte spheres to the axon cord periodically wrapped with the myelin sheath, as schematically depicted in Figure 6. The periodicity makes the chain similar to a 1D crystal. Despite that, the cord of an axon is a continuous ion tube. The modeling of it by the chain of segments defined by the periodic myelin sheath meets well with the plasmon-polariton kinetics which maintain its character the same in discrete chains and in continuous but periodically corrugated wires. The interaction between the chain elements (or segments defined by the myelin sheath) can be regarded as the dipole-type coupling. For chains of ionic spheres, one can adopt the results of the corresponding analysis for metallic chains, which support a dipole model of interaction between spheres $[21,29,30]$. Note also that the model of interacting dipoles [31,32] was originally developed for the description of ion stellar matter [33,34] and was then utilized for metal particle systems $[35,36]$. 

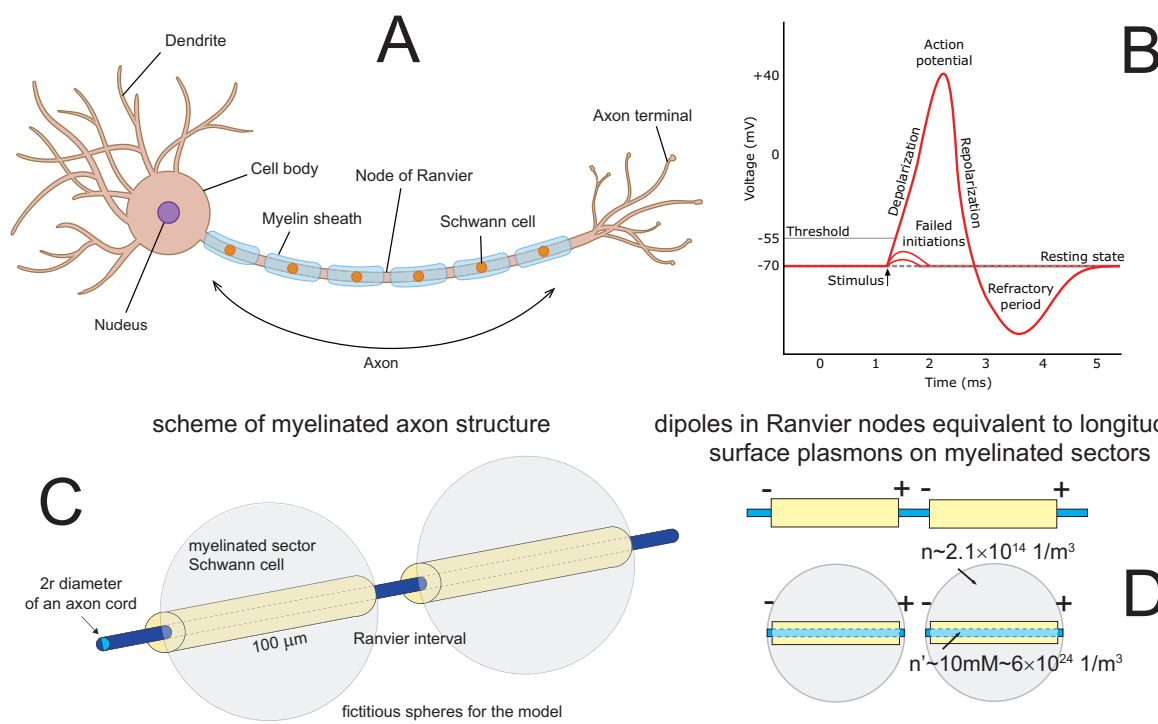

dipoles in Ranvier nodes equivalent to longitudinal surface plasmons on myelinated sectors

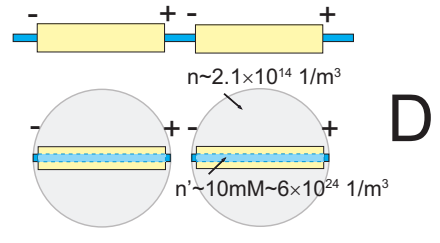

Figure 6. Schematic illustration of a long axon with a chain of periodically repeated myelinated sectors of approximately $100 \mu \mathrm{m}$ in length separated by unmyelinated Ranvier nodes, corresponding to a number of segments of order 10,000 per $1 \mathrm{~m}$ of axon length (A); time-pattern of the action potential forming on a Ranvier node (B); periodic fragments of a myelinated axon with a fictitious periodic chain of spherical ionic systems proposed as an effective model (C); the equivalence of polarized Ranvier nodes with longitudinal surface plasmons on myelinated sectors (effective concentration $n$ of ions in the auxiliary sphere corresponding to the actual ion concentration $\left.n^{\prime}\right)$ (D).

The dipole interaction resolves itself to the electric and magnetic fields created by an oscillating dipole $\mathbf{D}(\mathbf{r}, t)$ at any distant point. If this distant point is represented by the vector $\mathbf{r}_{0}$ (with one end fixed at the end of $\mathbf{r}$, where the dipole is placed), then the electric field produced by the dipole $\mathbf{D}(\mathbf{r}, t)$ has the following form, including the relativistic retardation $[8,22]$ :

$$
\begin{aligned}
& \mathbf{E}\left(\mathbf{r}, \mathbf{r}_{0}, t\right)=\frac{1}{\varepsilon}\left(-\frac{\partial^{2}}{v^{2} \partial t^{2}} \frac{1}{r_{0}}-\frac{\partial}{\partial \partial t} \frac{1}{r_{0}^{2}}-\frac{1}{r_{0}^{3}}\right) \mathbf{D}\left(\mathbf{r}, t-r_{0} / v\right) \\
& +\frac{1}{\varepsilon}\left(\frac{\partial^{2}}{v^{2} \partial t^{2}} \frac{1}{r_{0}}+\frac{\partial}{v \partial t} \frac{3}{r_{0}^{2}}+\frac{3}{r_{0}^{3}}\right) \mathbf{n}_{0}\left(\mathbf{n}_{0} \cdot \mathbf{D}\left(\mathbf{r}, t-r_{0} / v\right)\right),
\end{aligned}
$$

with $\mathbf{n}_{0}=\frac{\mathbf{r}_{0}}{r_{0}}$ and $v=\frac{c}{\sqrt{\varepsilon}}$ (here $\varepsilon$ denotes the dielectric susceptibility of the surroundings, with omitted subscript 1 for simplicity of notation). The terms with denominators of $r_{0}^{3}, r_{0}^{2}$, and $r_{0}$ usually are referred as to the near-field, medium-field, and far-field components of the interaction, respectively. The above formula allows for the description of the mutual interaction of the plasmon dipoles at each sphere in the chain. The spheres in the chain are numbered by integers $l$ and the equation for the surface plasmon oscillation of the $l$-th sphere can be written as follows ( $d$ denotes separation between sphere centers):

$$
\begin{aligned}
& {\left[\frac{\partial^{2}}{\partial t^{2}}+\frac{2}{\tau_{0}} \frac{\partial}{\partial t}+\omega_{1}^{2}\right] D_{\alpha}(l d, t)} \\
& =\varepsilon \omega_{1}^{2} a^{3} \sum_{m=-\infty, m \neq l}^{m=\infty} E_{\alpha}\left(m d, t-\frac{|l-m| d}{v}\right)+\varepsilon \omega_{1}^{2} a^{3} E_{L \alpha}(l d, t)+\varepsilon \omega_{1}^{2} a^{3} E_{\alpha}(l d, t),
\end{aligned}
$$

$\alpha=z$ indicates the longitudinal polarization, whereas $\alpha=x(y)$ the transverse polarization (the chain orientation is assumed to be along the $z$ direction). The first term on the right-hand side of Equation (39) describes the dipole coupling between spheres, and the other two terms correspond to the plasmon attenuation due to Lorentz friction-irradiation losses and the forcing field arising from an external electric field, respectively; $\omega_{1}=\frac{\omega_{p}}{\sqrt{3 \varepsilon}}$ is the frequency of the dipole surface plasmons. Ohmic losses are included via the term $\frac{2}{\tau_{0}}$ given by Equation (26) similar as applied to metal [37] but with the Fermi 
velocity of electrons substituted by mean velocity of ions for not degenerated classical Boltzmann distribution regardless of quantum statistics of ions.

According to Equation (38), we can write the following quantities that appear in Equation (39):

$$
\begin{aligned}
& E_{z}(m d, t)=\frac{2}{\varepsilon d^{3}}\left(\frac{1}{|m-l|^{3}}+\frac{d}{v|m-l|^{2}} \frac{\partial}{\partial t}\right) D_{z}(m d, t-|m-l| d / v), \\
& E_{x(y)}(m d, t)=-\frac{1}{\varepsilon d^{3}}\left(\frac{1}{|m-l|^{3}}+\frac{d}{v|m-l|^{2}} \frac{\partial}{\partial t}+\frac{d^{2}}{v^{2}|l-d|} \frac{\partial^{2}}{\partial t^{2}}\right) D_{x(y)}(m d, t-|m-l| d / v) .
\end{aligned}
$$

Because of the periodicity of the chain one can assume a wave-type collective solution of the dynamical equation in the form of Fourier component (39):

$$
\begin{aligned}
& D_{\alpha}(l d, t)=D_{\alpha}(k, t) e^{-i k l d}, \\
& 0 \leq k \leq \frac{2 \pi}{d} .
\end{aligned}
$$

In the Fourier picture of Equation (39) (the discrete Fourier transform (DFT) with respect to the positions and the continuous Fourier transform (CFT) with respect to time), this solution takes a form similar to that of the solution for the case of phonons in 1D crystals. Let us note that the DFT is defined for a finite set of numbers; therefore, we consider a chain with $2 N+1$ spheres, i.e., a chain of finite length $L=2 N d$. Then, for any discrete characteristic $f(l), l=-N, \ldots, 0, \ldots, N$ of the chain, such as a selected polarization of the dipole distribution, one must consider the DFT picture $f(k)=\sum_{l=-N}^{N} f(l) e^{i k l d}$ where $k=\frac{2 \pi}{2 N d} n, n=0, \ldots, 2 N$. This means that $k d \in[0,2 \pi)$ because of the periodicity of the equidistant chain. The Born-Karman periodic boundary condition, $f(l+L)=f(l)$, is imposed on the entire system, resulting in the form of $k$ given above. For a chain of infinite length, one can take the limit $N \rightarrow \infty$, which causes the variable $k$ to become quasi-continuous, although $k d \in[0,2 \pi)$ still holds.

The Fourier representation of Equation (39) has the following form:

$$
\begin{aligned}
& \left(-\omega^{2}-i \frac{2}{\tau_{0}} \omega+\omega_{1}^{2}\right) D_{\alpha}(k, \omega) \\
& =\omega_{1}^{2} \frac{a^{3}}{d^{3}} F_{\alpha}(k, \omega) D_{\alpha}(k, \omega)+\varepsilon a^{3} \omega_{1}^{2} E_{0 \alpha}(k, \omega),
\end{aligned}
$$

with

$$
\begin{aligned}
& F_{z}(k, \omega)=4 \sum_{m=1}^{\infty}\left(\frac{\cos (m k d)}{m^{3}} \cos (m \omega d / v)+\omega d / v \frac{\cos (m k d)}{m^{2}} \sin (m \omega d / v)\right) \\
& +2 i\left[\frac{1}{3}(\omega d / v)^{3}+2 \sum_{m=1}^{\infty}\left(\frac{\cos (m k d)}{m^{3}} \sin (m \omega d / v)-\omega d / v \frac{\cos (m k d)}{m^{2}} \cos (m \omega d / v)\right)\right], \\
& F_{x(y)}(k, \omega)=-2 \sum_{m=1}^{\infty}\left(\frac{\cos (m k d)}{m^{3}} \cos (m \omega d / v)\right. \\
& \left.+\omega d / v \frac{\cos (m k d)}{m^{2}} \sin (m \omega d / v)-(\omega d / v)^{2} \frac{\cos (m k d)}{m} \cos (m \omega d / v)\right) \\
& -i\left[-\frac{2}{3}(\omega d / v)^{3}+2 \sum_{m=1}^{\infty}\left(\frac{\cos (m k d)}{m^{3}} \sin (m \omega d / v)\right.\right. \\
& \left.\left.+\omega d / v \frac{\cos (m k d)}{m^{2}} \cos (m \omega d / v)-(\omega d / v)^{2} \frac{\cos (m k d)}{m} \sin (m \omega d / v)\right)\right] .
\end{aligned}
$$

Similarly as in the case of metallic nano-chains [38], $\operatorname{Im}_{\alpha}(k, \omega) \equiv 0$ (for $\alpha=z, x(y)$ ), which indicates the perfect quenching of the irradiation losses at any sphere in the chain (it means that to each sphere the same amount of energy incomes from other spheres as outflows due to the Lorentz friction). One can easily verify this property as the related infinite sums in Equation (43) can be found analytically $[38,39]$. Equation (42) is highly nonlinear with respect to the complex $\omega$ and can be solved both perturbatively in the analytical manner [27] or numerically-more accurately [40]. The determined solutions for Re $\omega$, Im $\omega$ (i.e., for self-frequency and damping of plasmon-polariton) can be applied to the model of axon as the effective chain of electrolyte spheres with adjusted ion concentration to the real neuron parameters. 
The problem of propagation of the plasmon-polaritons along metallic nano-chains was developed, among others, by Citrin [25,41] in order to assess the radiative losses of plasmon-polariton in metallic nano-chains in agreement with former observations related to radiation losses in one-dimensional and two-dimensional crystals [42]. The resonance frequency $(\operatorname{Re} \omega(k))$, its $k$-derivative, i.e., the group velocity and the attenuation rate $(\operatorname{Im} \omega(k))$ of dipole plasmon-polariton modes numbered by the wave vector $k$, derived by solution of Equation $(42)[27,40]$ for ionic chains with exemplary concentration, chain size and ion parameters are plotted in Figure 7.
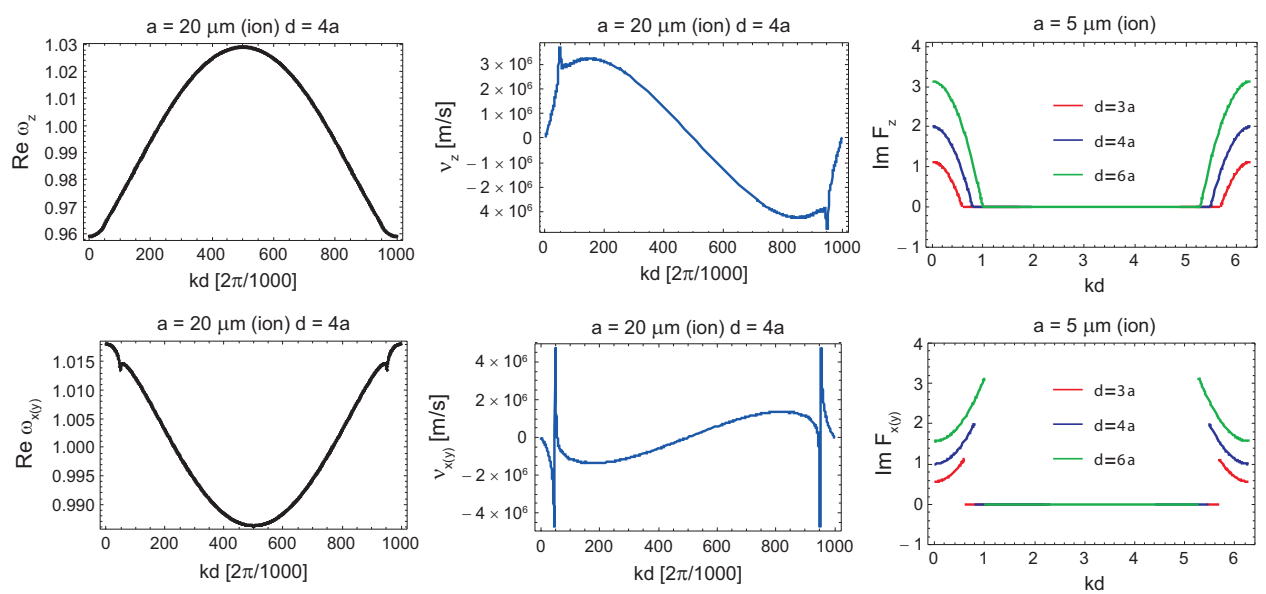

Figure 7. The exact solutions for the self-frequencies of the longitudinally and transversely polarized modes of plasmon-polaritons in an ionic chain ( $\omega$ in units of $\left.\omega_{1}\right)$ obtained by exactly solving Equation (42) at 1000 points in the region $k d \in[0,2 \pi)$ (left) and the corresponding group velocities for both types of polarization (center); the functions $\operatorname{ImF}_{z}\left(k ; \omega=\omega_{1}\right)$ and $\operatorname{Im} F_{x(y)}\left(k ; \omega=\omega_{1}\right)$ for infinite chains of electrolyte spheres of radius $a$ at separations of $d=3 a, 4 a$, and $6 a$, the shift of the singularities toward band edges with lowering $d / a$ is noticeable (right).

\section{Plasmon-Polariton Model of Saltatory Conduction-Fitting of Plasmon-Polariton Kinetics to Axon Parameters}

The bulk plasmon frequency $\omega_{p}=\sqrt{\frac{q^{2} n 4 \pi}{m}}$ (we assumed for the model the ion charge $q=$ $1.6 \times 10^{-19} \mathrm{C}$ and the ion mass $m=10^{4} m_{e}$, where $m_{e}=9.1 \times 10^{-31} \mathrm{~kg}$ is the mass of an electron) and for $n=2.1 \times 10^{14} 1 / \mathrm{m}^{3}$ concentration we obtain the Mie-type frequency for ionic dipole oscillations, $\omega_{1} \simeq 0.1 \frac{\omega_{p}}{\sqrt{3 \varepsilon_{1}}} \simeq 4 \times 10^{6} 1 / \mathrm{s}$, where the relative permittivity of water is $\varepsilon_{1} \simeq 80$ for frequencies in the $\mathrm{MHz}$ range [43] (although for higher frequencies, beginning at approximately $10 \mathrm{GHz}$, this value decreases to approximately 1.7, corresponding to the optical refractive index of water, $\eta \simeq \sqrt{\varepsilon_{1}}=1.33$ ). For the thin axon cord and thus, in the discrete model, strongly prolate inner ionic cord segment, we reduce the longitudinal Mie-type frequency by a factor of 0.1 [44,45] compared with the isotropic spherical case. Let us emphasize that the axon consists of a cord with a small diameter of $2 r$, and this thin cord is wrapped with a myelin sheath of a length of $2 a$ per segment; however, for the effective model, we consider fictitious electrolyte spheres of radius $a$. Thus, the auxiliary concentration $n$ of ions in the fictitious spheres corresponds to a concentration of ions in the cord of $n^{\prime}=\frac{n 4 / 3 \pi a^{3}}{2 a \pi r^{2}}$, which yields a typical concentration of ions in a nerve cell of $n^{\prime} \sim 10 \mathrm{mM}$ (i.e., $\sim 6 \times 10^{24} 1 / \mathrm{m}^{3}$ ). This is because all ions participating in the dipole oscillation correspond in the sphere model to a much smaller volume in the real system, that of the thin cord portion (the insulating myelinated sheath consists of a lipid substance without any ions). The insulating, relatively thick myelin coverage creates the periodically broken channel (corrugated conductor-insulator structure) required for plasmon-polariton formation and its propagation. To reduce the coupling with the surrounding inter-cellular electrolyte and protect against any leakage of the plasmon-polaritons, the myelin sheath must be sufficiently thick, much thicker than what is required merely for electrical insulation. Moreover, to accommodate the conductivity parameters that we calculated for a spherical geometry to the highly prolate geometry 
of the real oscillating ionic system, we must account for the fact that the Mie-type frequency of the longitudinal oscillations must be significantly lower than that for a sphere with a diameter equaled to the elongation axis. As a rough estimate, we assumed a correction factor of 0.1 [44,45].

For the resulting Mie-type frequency, $\omega_{1} \simeq 4 \times 10^{6} 1 / \mathrm{s}$, one can determine the plasmon-polariton self-frequencies in a chain of spheres of radius $a=50 \mu \mathrm{m}$ (for a Schwann cell length of $2 a$ ) and for small chain separations of $d / a=2.01,2.1$, and 2.2 (corresponding to Ranvier node lengths of $0.5,5$, and $10 \mu \mathrm{m}$, respectively) within the approach presented above (via solution of Equation (42)). The derivative of the obtained self-frequency with respect to the wave vector $k$ determines the group velocity of the plasmon-polariton modes. The results for the model system (as in Figure 6) are presented in Figure 8. We observe that for the ionic system parameters listed above, the group velocity of the plasmon-polaritons reaches $100 \mathrm{~m} / \mathrm{s}$ for longitudinal mode with polarization suitable to the prolate geometry assuming that the initial post-synaptic action potential or that from the synapse hillock excites predominantly longitudinal ion oscillations.
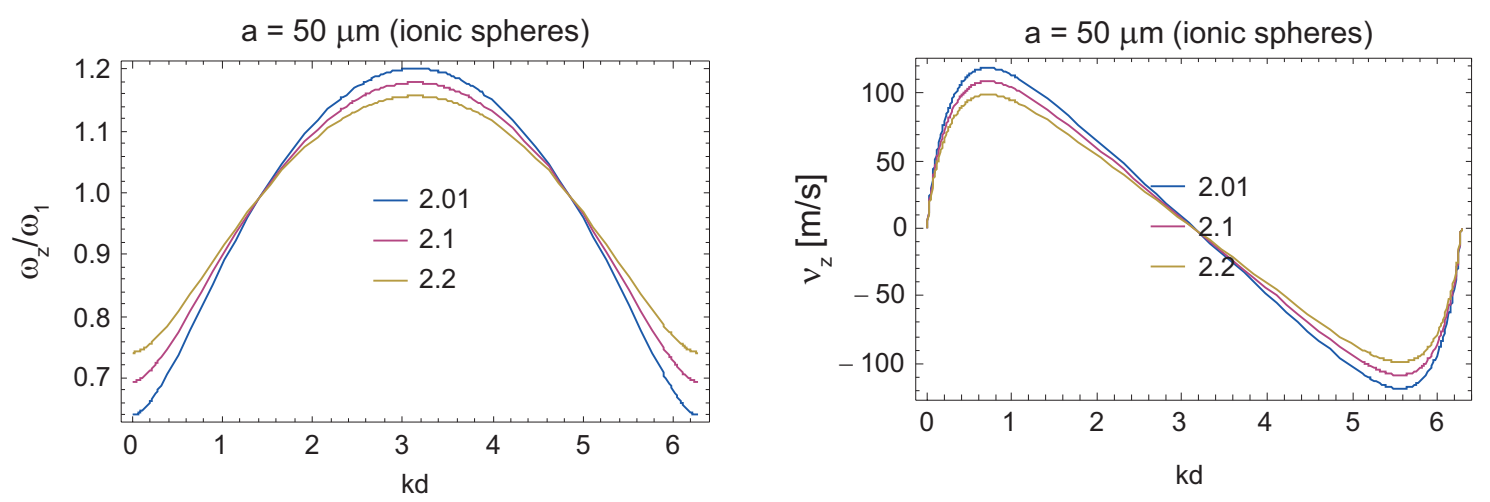

Figure 8. Solutions for the self-frequencies and group velocities of the longitudinal mode of a plasmon-polariton in the model ionic chain; $\omega$ is presented in units of $\omega_{1}$, here $\omega_{1}=4 \times 10^{6} 1 / \mathrm{s}$, for a chain of spheres of radius $a=50 \mu \mathrm{m}$ and a separation of $d / a=2.01,2.1$, or 2.2 for an equivalent ion concentration in the inner ionic cord of the axon of $n^{\prime} \sim 10 \mathrm{mM}$.

Note that for $\omega_{1}=4 \times 10^{6} 1 / \mathrm{s}$ and $a=50 \mu \mathrm{m}$, the light-cone interference conditions $k d-\omega_{1} d / c=$ 0 and $k d+\omega_{1} d / c=2 \pi$ are fulfilled for extremely small values of $k d$ and $2 \pi-k d$, respectively (of the order of $10^{-6}$ for $\left.d / a \in[2,2.5]\right)$, and thus are negligible with regard to the plasmon-polariton kinetics under these conditions (though were important for metals). The related singularities on light-cone induced by the far- and medium-field contributions to the dipole interaction are pushed to borders of the $k$ wave vector domain and thus are unimportant in the case of considered ionic system. Hence, the quenching of the radiative losses (i.e., the perfect balance the Lorentz friction in each sphere by the radiation income from other spheres in the chain) for the plasmon-polariton modes in the axon model occurs practically throughout the entire $k d \in[0,2 \pi)$ region. Additionally, the above-mentioned singularities [40] are characteristic of infinite chains and therefore cannot fully develop because the nerve model electrolyte chains are of a finite length, whereas other effects, as quenching of irradiation losses, occur for finite chains due to very fast convergence of sums in Equation (43) with denominators $\mathrm{m}^{2}$ and $\mathrm{m}^{3}$ (practically, the chain consisting of only 10 elements exhibits almost same properties as the infinite one).

Although the ionic system chain model for a myelinated axon appears to be a crude approximation of the real axon structure, it can serve for the comparison of the energy and time scales of plasmon-polariton propagation implied by the model with the observed kinetic parameters of nerve signals. In the model, the propagation through the axon chain of a plasmon-polariton that is excited by an initial action potential on the first Ranvier node (after the synapse or, for the reverse signal direction, in the neuron cell hillock) sequentially ignites consecutive Ranvier node blocks of $\mathrm{Na}^{+}$and $\mathrm{K}^{+}$ion gates, and the resulting firing of the action potential traverses the axon with an observed 
velocity of approximately $100 \mathrm{~m} / \mathrm{s}$, consistent with the velocity actually observed in myelinated axons (and not possible for ionic diffusive current upon the cable model $[9,12])$. The plasmon-polariton ignition of consecutive Ranvier nodes releases creation of the same activation potential pattern aided by the external energy supply at each Ranvier node block. Because of the nonlinearity of ion-channel block mechanism, the signal growth saturates at a constant level, and the overall timing of each action potential spike has the stable shape of the local polarization/depolarization scheme in the short fragment of cell membrane that corresponds to the non-myelinated Ranvier node. The permanent supply of energy associated to the creation of the action potential spikes at one-by-one firing Ranvier nodes contributes also to the plasmon-polariton dynamics assuring the amplitude of each Ranvier block dipole excitation is beyond the activation threshold. The external energy supply (through the conventional ATP / ADP cell mechanism) assisting the renovation of the action potential pattern at each Ranvier node residually compensates the Ohmic thermal losses of the plasmon-polariton mode propagating along the axon, and ensures that its propagation is undapmed over an unlimited range. Although the entire signal cycle of the action potential on a single Ranvier node block requires several milliseconds (or even longer when one includes the time required to restore the steady-state conditions, which, on the other hand, conveniently blocks the reversing of the signal), subsequent nodes are ignited more rapidly, corresponding to the velocity of the plasmon-polariton wave packet triggering the ignition of the consecutive Ranvier nodes, as illustrated in Figure 9. Thus we deal with the firing of the axon which propagates with the velocity of the ionic plasmon-polariton wave packet. The direction of the velocity of the plasmon-polariton wave packet is adjusted to the semi-infinite geometry of the chain (in fact, the chain is finite and is excited at one its ends). The firing of the action potential that is triggered by the plasmon-polariton traverses along the axon in only one direction because the nodes that have already fired have had their $\mathrm{Na}^{+}, \mathrm{K}^{+}$gates inactivated (discharged) and will require a relatively long time to restore these gates to their original status (the entire sodium/potassium block requires time of the order of even a second as well as a sufficient energy supply to bring the concentrations to their normal values via cross-membrane active ion pumps against the ion-density gradient). The described above plasmon-polariton scheme for the ignition of the action potential spikes in the ordered chain of Ranvier nodes along the axon is thus well consistent with the saltatory conduction observed in myelinated axons. Observation that firing of the action potential can move in two opposite directions simultaneously if one ignites some central Ranvier node of a passive axon, as well as the observation of the maintenance of the firing traverse despite small breaks of the axon cord or damage of few Ranvier nodes, also agree with the collective wave-type plasmon-polariton model of the saltatory conduction, in contrast to the lack of a satisfactory explanation in models based on the cable theory. The maintenance of the plasmon-polariton kinetics, despite discontinuity of the axon cord, well agrees with the discrete chain model of the cord corrugated by the periodic myelin sheath.

In Figure 10, the group velocity of the action potential traversing a firing myelinated axon (in plasmon-polariton model) is plotted for various diameters of the internal cord of the axon, with a length of $100 \mu \mathrm{m}$ for each myelinated sector wrapped by Schwann cells, and Ranvier intervals of $0.5 \mu \mathrm{m}, 5 \mu \mathrm{m}$, and $10 \mu \mathrm{m}$. The dependence on the length of the Ranvier interval is weak (i.e., negligible at the considered scale in consistence with the equivalence of the discrete model for the continuous system if one considers the wave type plasmon-polariton propagation), but the increase in the velocity with increasing thickness of the internal cord is significant, similarly as in the real axons with growing diameter. 
Firing of the myelinated axon by propagation of the plasmon polariton wave packet

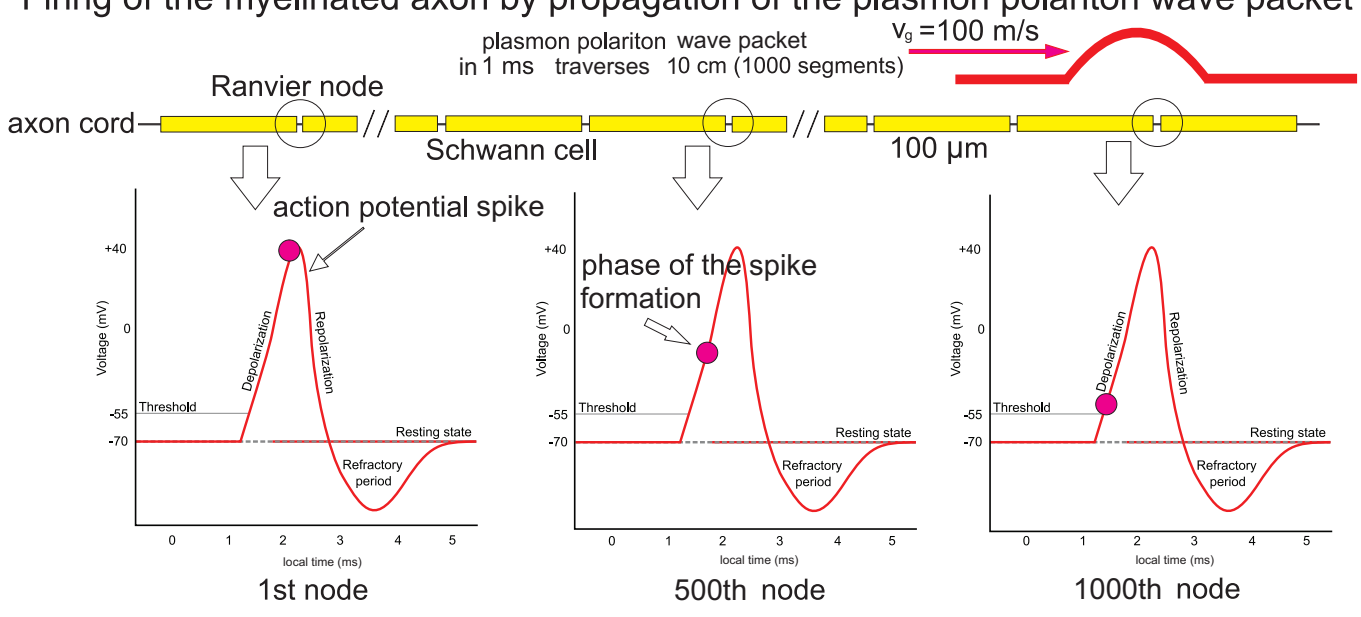

$1 \mathrm{~ms}$ of propagation of plasmon polariton triggers in sequence 1000 Ranvier nodes on the distance of $10 \mathrm{~cm}$

Figure 9. Schematic presentation of the firing of a nerve cell triggered by the propagation of the plasmon-polariton wave packet along the periodic structure of the myelinated axon; for group velocity of the plasmon-polariton wave packet of order of $100 \mathrm{~m} / \mathrm{s}$ within $1 \mathrm{~ms}$ the packet traverses $10 \mathrm{~cm}$ distance and initiates one by one the forming of the action potential on 1000 in sequence Ranvier nodes being thus in various time phases as indicated by red dots for exemplary nodes.
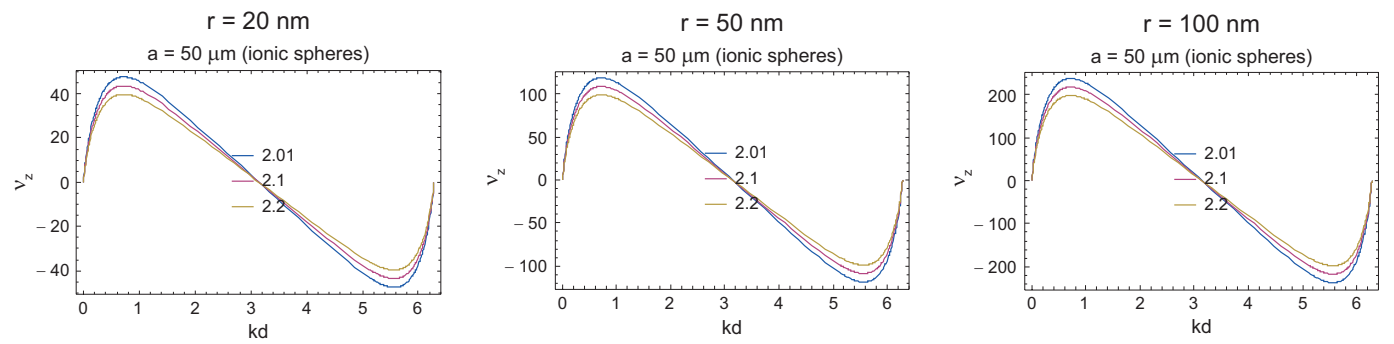

Figure 10. Comparison of the group velocities, in units of $\mathrm{m} / \mathrm{s}$, of the longitudinal plasmon-polariton mode with respect to the wave vector $k \in[0,2 \pi / d)$ within the axon model for a Schwann cell myelinated sectors with a length of $100 \mu \mathrm{m}$, Ranvier separations of $0.5 \mu \mathrm{m}, 5 \mu \mathrm{m}$, and $10 \mu \mathrm{m}$ (represented by $d / a=2.01,2.1$, and 2.2 in the figure, respectively) and for the axon cord radii of $r=20,50$, and $100 \mathrm{~nm}$.

To comment on the appropriateness of the chain model to the axon case let us note that even though the thin core of the axon is a continuous ion conducting fiber, the surface electromagnetic field can be closely pinned to the linear conductor similarly as for the Goubau line (well-known from the micro-wave technology) $[46,47]$ and if periodically wrapped by dielectric shells, the plasmon resonantly coupled with the EM field propagates as much more concentrated plasmon-polariton along the chain of periodic segments despite continuity of the fiber. For plasmon-polariton kinetics the continuity of the conducting fiber is unimportant because we deal here with traversing of the wave packet of the plasma oscillation and not of charge carriers themselves, similarly as also in Goubau micro-wave lines also with discontinuous segments. The Goubau lines maintain their transmittance via discrete, unconnected elements. The fragments of the thin axon cord wrapped with the myelin thick shells with typical for neurons ion concentration $n^{\prime} \simeq 10 \mathrm{mM} \simeq 6 \times 10^{24} 1 / \mathrm{m}^{3}$ inside the cell, can be equivalently modeled by the sphere with diameter equaled to the length of cord fragment and with the ion concentration $n \simeq 2 \times 10^{14} 1 / \mathrm{m}^{3}$ (for the axon cord diameter assumed to be $100 \mathrm{~nm}$ ) conserving the number of ions in the segment taking part in the dipole oscillations. Such a model is justified by the same structure of the dynamics equation for dipole plasmon fluctuations in the chain of spheres, i.e., of Equation (39), and of its modification for the prolate spheroid or elongate cylindrical rod chain. This modification resolves itself to the substitution in Equation (39) of isotropic $\omega_{1}$ frequency by frequencies different for 
each polarization $\omega_{\alpha 1}$, i.e., $\left[\frac{\partial^{2}}{\partial t^{2}}+\frac{2}{\tau_{\alpha}} \frac{\partial}{\partial t}+\omega_{\alpha 1}^{2}\right] D_{\alpha}(l d, t)=\mathcal{A} \sum_{m=-\infty, m \neq l}^{m=\infty} E_{\alpha}\left(m d, t-\frac{|l-m| d}{v}\right)$

$+\mathcal{A} E_{L \alpha}(l d, t)+\mathcal{A} E_{\alpha}(l d, t)$, where $\mathcal{A}=V \frac{n q^{2}}{m}$ is a shape independent factor proportional to the number of ion carriers with concentration $n$ in the volume of the spheroid with semi-axes $a, b, c, V=\frac{4 \pi}{3} a b c=$ $\frac{4 \pi}{3} a^{3}$ (the latter for a sphere), cf. Figure 11. Taking into account that the plasmon frequency in bulk electrolyte with ion concentration $n$ equals to $\omega_{p}=\sqrt{\frac{n q^{2} 4 \pi}{m}}$, one can rewrite $\mathcal{A}$ as follows, $\mathcal{A}=\frac{a b c \omega_{p}^{2}}{3}=\varepsilon a^{3} \omega_{1}^{2}$ (the latter for a sphere, for which $\omega_{1}=\frac{\omega_{p}}{\sqrt{3 \varepsilon}}$ ). Ohmic losses can be included via the anisotropic term, $\frac{1}{\tau_{\alpha 0}}=\frac{v}{2 \lambda_{B}}+\frac{C v}{2 a^{\alpha}}$, where $a^{\alpha}$ is the dimension (semi-axis) of the spheroid in the direction $\alpha$ (equaled to $a, b, c$ for a spheroid). The first isotropic term in the expression for $\frac{1}{\tau_{\alpha 0}}$ approximates ion scattering losses such as those occurring in the bulk electrolyte (thus is isotropic), whereas the second term describes the losses due to the scattering of ions on the anisotropic boundary of the spheroid. Actually this term can be neglected for the longitudinal polarization because the neuron cord in continuous along the $z$ direction. The dipole coupling is independent of the shape of chain elements. The above described mutual independence of dipole oscillations with distinct polarizations follows from the linearity of the dynamics equation regardless the metal or electrolyte conducting elements.

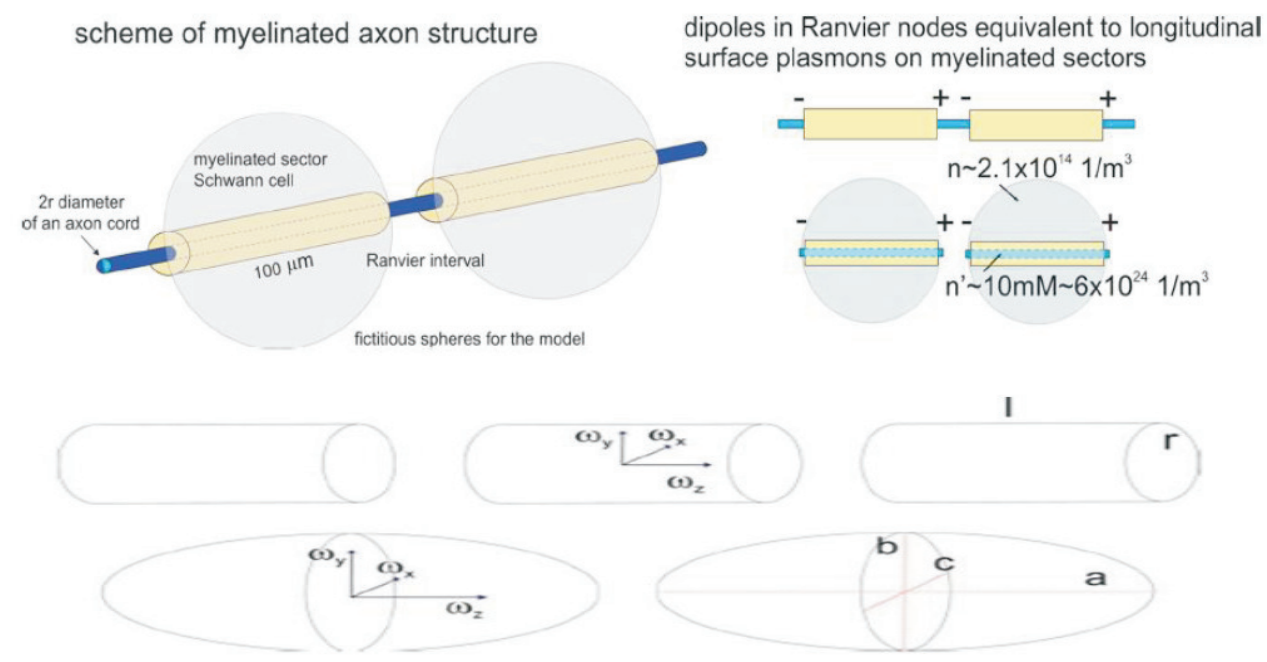

Figure 11. In the effective chain-model of the continuous axon corrugated by periodic myelin sectors corresponds rather to a series of rods or prolate spheroids; because of linearity with respect to the dipole field of the dynamic equation it is possible to renormalize equations for both polarizations without changing their structure.

Because the structure of the dynamics equation is not affected by the anisotropy, thus its solutions for each polarization have the same form as in the spherical case with the exception to the modification of the related frequency of the dipole oscillations in each direction and the small correction to the orientation dependent contribution to the scattering ratio (this part being related to the boundary scattering of carriers, not important for longitudinal polarization when in fact the axon cord is continuous). One can thus renormalize the equation for dipole oscillations independently for each polarization direction, introducing in a phenomenological manner the resonance oscillation frequency for each direction $\omega_{\alpha 1}$ (these can be estimated numerically, whereas for a sphere $\omega_{1}=\frac{\omega_{p}}{\sqrt{3 \varepsilon}}$; in general, the longer semi-axis the lower related dipole oscillation frequency is). Moreover, the size-correction of the boundary scattering term must be taken into account as described above (favorably reducing of longitudinal plasmon-polariton damping for the prolate geometry). Such a renormalization was done for a considered model of the axon segment chain.

The periodic structure of the myelinated axon does not form a chain of electrolyte spheres, but rather is a thin electrolyte cord wrapped in myelin sheath on periodically distributed sectors separated 
by very short unmyelinated intervals-Ranvier nodes. The periodic corrugated structure of the dielectric isolation allows, however, for consideration of collective plasmon wave-type oscillations, $\sim e^{i} q z$ with $q$ governed by the periodicity, propagating along the cord which resolve themselves to wave-type synchronic dipole polarization of consecutive Ranvier intervals. Taking into account that Ranvier nodes are short, these polarization dipoles are equivalent with similarly synchronically oscillating dipoles of myelinated sectors. The latter can be thus treated as dipole surface plasmon oscillations propagating along the chain of prolate spheroids with small separations and with the longitudinal dipole polarization, despite actually continuous character of the cord-this is sketched in Figure 6 (right panel). To estimate characteristics of such collective dipole wave-type excitation along the cord sectors one can use the model of the chain of spherical electrolyte systems with suitably diluted ion concentration (resulted in the same total ion number as in the cord fragments) and with the decreased resonance frequency as for the longitudinal polarization in the case of highly prolate spheroid (or elongated cylinder). The model allows the quantitative estimation of the relevant propagation characteristics and the verification whether plasmon-polariton dynamics might fit to the observed features of the saltatory conduction in myelinated axons, being mysterious and obscure as of yet.

Plasmon-polariton wave-packet triggers at consecutive Ranvier nodes the release of ions through the non-myelinated membrane and the formation of the action potential (its renewal at each Ranvier node). The relatively low polarization induced by plasmon-polariton initiates at a Ranvier node opening of the $\mathrm{Na}^{+}$and $\mathrm{K}^{+}$across-membrane ion channels, which results in a characteristically large action signal due to the transfer of ions through the opened gates caused by the difference in their concentrations on either sides of the membrane. The entire cycle requires a few milliseconds, but the initial increase in polarization caused by the rapid opening of the $\mathrm{Na}^{+}$channel occurs on the scale of a single millisecond. Because the myelin layer wrapped by the Schwann cell prohibits cross-membrane ion transfer, the local polarization/depolarization of the internal cytoplasm of the axon takes place only at the Ranvier nodes, thus strengthening the dipole formation in the axon fragment wrapped by the Schwann cell and strengthening the amplitude ot the wave-type collective dipole oscillations in the whole chain. Each Ranvier node activity triggered by the plasmon-polariton one-by-one lifts the plasmon-polariton amplitude to the constant value despite the Ohmic losses. The plasmon-polariton mode that traverses the axon structure consisting of periodically polarized electrolyte segments wrapped with myelin sheath cause the consecutive individual ignitions of the Ranvier nodes in sequence along the axon. This triggering role of the plasmon-polariton elucidates thus how the action signal jumps between neighboring active Ranvier nodes.

The plasmon-polariton scenario in an ionic chain, even in the simplified version, might share certain features in common with the ability of nerve systems to achieve very efficient and energetically economical electric signaling despite the rather poor ordinary electric conductivity of axons. The plasmon-polariton kinetics in the periodic structure of the axon may be a convenient explanation for high performance nerve signaling by providing quick signal propagation despite low ordinary conductivity for the practically unlimited range when the energy is persistently supplied to cover only relatively small plasmon-polariton Ohmic losses (lower than for ordinary current). Moreover, plasmon-polaritons do not irradiate any energy. The energy supply is provided residually by the ATP / ADP mechanism in neuron cell, which energetically contributes to the signal-dependent opening and closing of $\mathrm{Na}^{+}$and $\mathrm{K}^{+}$channels in the Ranvier nodes and then to the restoration of the steady-state conditions, i.e., via the active transport of ions across the membrane against the ionic concentration gradient which needs the external energy supply. Moreover, the coincidence of the micrometer scale of the axon's periodic structure of Schwann cells (of approximately $100 \mu \mathrm{m}$ in length) with the typical requirements for the size of ionic chains supports the suggested plasmon-polariton concept as the explanation of the transduction of the action potential along the axon in agreement with the tentative quantitative fitting of the model. 
It must also be emphasized that plasmon-polaritons do not interact with external electromagnetic waves, or, equivalently, with photons (even with adjusted frequency), which is a consequence of the large difference in the group velocity between the plasmon-polariton and the photon velocity $(c / \sqrt{\varepsilon})$. The resulting large discrepancy in wavelength between the photon and the plasmon-polariton of the same energy prohibits the mutual transformation of these two types of excitations because of momentum-energy conservation constraints. Therefore, plasmon-polariton signaling by means of collective wave-type dipole plasmon oscillations along a chain-plasmon-polariton-can be neither detected nor perturbed by the external electromagnetic radiation. This also well fits with neural signaling properties in the peripheral nervous system and in the white myelinated matter in the central system. It is also worth noting that the ionic surface plasmon frequencies are independent of temperature, although the ionic volume plasmon frequencies are temperature dependent, as has been proved in the former paragraph. However, the temperature influences the mean velocity of the ions, $v=\sqrt{\frac{3 k T}{m}}$, thereby causing an enhancement in Ohmic losses with increasing temperature (cf. Equation (26)), which, in turn, strengthens the plasmon-polariton damping. Hence, at higher temperatures, higher external energy supplementation is required to maintain the same long-range propagation of plasmon-polaritons with a constant amplitude. This property is also consistent with experimental observations. Note, however, that energy costly is an active ion transport through the membrane to recover the steady state of each of Ranvier nodes. Only residual part of this energy is sufficient to eventually support and maintain undamped plasmon-polariton propagation, which can be regarded in the scheme of the steady solution of the forced and damped oscillator applied here to plasmon-polariton dynamics similarly as in the metallic chain activated by a coupled external source (e.g., a coupled quantum dot system [48]).

Note that the energy supply from the consecutive excited Ranvier nodes continuously covering plasmon-polariton Ohmic losses results also in a convenient narrowing of the plasmon-polariton $k$-wave packet shifting it toward the long-wave limit (small $k$ ), which follows from the Fourier picture of the Dirac delta (the larger number of the chain segments contributes to the excitation of the plasmon-polariton, the narrower plasmon-polariton $k$-wave packet is formed). This favorable property allows for the precise ignition of sequential Ranvier nodes by unlimitedly propagating relatively narrow plasmon-polariton wave packet triggering consecutive Ranvier nodes.

The utilization of the plasmon-polariton propagation irradiatively undamped in the chain of electrolyte subsystems may lead to an explanation of the efficient and long range so-called saltatory conduction in myelinated axons in the peripheral neural system and in the white matter of brain and spinal cord. The effective plasmon-polariton model of the triggering of action potential firing along the axon in peripheral nervous system myelinated by Schwann cells separated with Ranvier nodes fits well with the observed large conduction velocity and its temperature and size dependence. This coincidence together with the immunity of plasmon-polariton to the external EM perturbation or detection support the reliability of the proposed new model for the saltatory conduction of action potential, which is very efficient and energy frugal despite the poor ordinary conductivity of axons.

\section{Soft Plasmonics Application toward the Recognition of the Role of Gray and White Matter in Information Processing}

The electric activity of nervous system is referred to transduction of action potential spike series along axons and dendrites of neuron cells connected together in electro-chemical synapses into a highly entangled web. Not all trajectories for signal travel are accessible (blocked by passive synapses or by their absence) and the activation of selected synapses makes traces available to communication for longer or shorter time-periods depending on intensity and repetition of the synapse activation. Via the activation of selected (even in a random manner) synapses it creates an entangled pattern or a braided web of trajectories. The role of braids seems to be more important than only simple connections (addressing) in such an activated neuron network. The classification of the resulted entanglement patterns may be done in topological terms of so-called pure braid groups [49] as will be 
demonstrated in the next paragraph. Elements of these groups display various inequivalent patterns of entanglement of treads (neuron filaments) allowing, however, a precise comparison and distinguishing of complicated filament muddles in a mathematically rigorous topological-homotopy way. Two braids are homotopic if one of them can be transformed into another one by continuous deformations without cutting. For $N$ treads the number of various topologically inequivalent patterns of braids is infinite though countable [49], in practice limited only by size restrictions, like a finite diameter and length of filaments. The variety of various patterns-braids highly exceeds the ordinary addressing which for $N$ filament gives only $N$ ! opportunities, low in comparison to infinite number of $N$ filament braids.

If one activates electrically such an entangled network, pushing an AC charge current along its treads, one can notice that the unique pattern of braiding with a specific number of loops might serve as multiloop EM active circuit-the distributed coil. The AC electric current in such braid-coil would generate thus an individual also unique local-space EM field distribution which could fall into resonance with another web part representing the same braid group element located within the range of this dynamic field distribution. One can associate thus information messages (elements of the memory) imprinted in distinct elements of the pure braid group implemented in a nonlocal manner in fragments of the large neuron web of the brain via previous activation (creation) and temporarily consolidation of some subset of synapses. This massage could be temporarily stored in the neuron-braid corresponding to some mathematical braid from the pure braid group as long as the selected engaged synapses are still active (or are ready to be active). This information can be next invoked by EM resonance with the similar braid within the brain and temporarily created in distant its structure (e.g., in the hippocampus) as the result of an influx of neuron signals from surroundings via senses. The new message tentatively imprinted in a temporary braid in the hippocampus might excite via the EM resonance the same braid recorded in the past in the cortex, and in this way may cause the identification of a new message in the mind. Let us note here that dendrites in the gray matter have prepared thousands of lateral spikes ready to create new synapses between neurons.

To utilize the braids in the proposed EM recognition process, the electrical AC currents are necessary to initiate a resonance response. The role of such AC-electricity may play brain waves which actually are constantly observed via EEG in the gray matter of the cerebral cortex. For transduction of neuron signals in non-myelinated axons and in all dendrites in the cortex, the diffusive movement of ions in elongate and branched neuron cells is employed. This electric-current-type communication is very well described by the cable theory originated by W. Thompson (1854) [10] and widely applied to the explanation of the neural electro-physiology of dendrites and non-myelinated axons (with various modifications $[11,50])$.

Hence, one can expect that the storage and the identification of the information take place in the cerebral cortex taking an advantage of the huge information capacity of entanglement of neuron filaments creating various braid structures enumerated by the pure braid group elements. The topologically precise homotopy-resolution and a quick and complex access to the stored messages in entangled braids [49,51-53] via the EM resonance utilizing additionally a few distinct frequencies of the brain waves might be helpful to better understand the functionality of the memory and the pattern recognition by a mind. All these take place in the gray matter of the cortex where the signal transduction is carried by means of the diffusive movement of ions equivalent to an ordinary electric AC current, which is able to induce local EM field in a coil. Different types of frequencies of the brain waves might be here convenient to implement simultaneous independent resonance channels for better identification of multiloop coils created by neuron filament braids [53].

The braid groups have been introduced to describe entangled bundles of classical trajectories of $N$ particle systems [49]. Two types of braid groups are investigated and applied, the first one with the assumption of indistinguishability of identical particles, called the full braid groups and applied to characterize quantum statistics of particles [52] and the second type-the so called pure braid groups for ordinary distinguishable particles [49,52]. The latter are suitable to describe and classify highly entangled multi-filament webs. Both full and pure braid groups are infinite for $N$ particles 
moving on the plane but are finite groups when particles move in 3D space. In 3D, there is room to disentangle lines representing particle trajectories, what is, however, impossible in 2D (in 2D particle must twist up one around another and cannot hop beyond or beneath others). The pure braid group of $N$ distinguishable particles on a plane (2D) may be presented as the set of $N$ treads in 3D with their ends fixed to steady points but braided on the way in an arbitrary manner. Thus the 3D web of entangled treads as of neurons in a brain can be mapped on 2D pure braid group. The patterns of such braids are unique and cannot be transferred one into another one without cuttings of treads and can be precisely enumerated by the pure braid group elements. If one imagines the $N$ initial and $N$ final points of treads steady fixed in 3D space, then the structure of linking them braids of threads-the 2D $\mathrm{N}$-element pure braid group elements reproduces one-to-one 3D webs of arbitrary entangled nets of filaments, like a webs of neurons linked via selected synapses in the brain.

\subsection{Possible Linkage with the Topological Model of Neuron Web}

The topology characteristics of entangled filament webs, like neuron webs, may be described in terms of homotopy reflecting the complexity of trajectories of many particle systems [54]. The first homotopy group of the space $D$ marked with $\pi_{1}(D)$ is the collection of topologically non-equivalent (non-homotopic, i.e., one cannot be continuously deformed to another) classes of closed trajectories in the space $D$. If this space is a configuration space of a system of $N$ particles, each of which runs its own trajectory on a manifold $M$, then the appropriate $\pi_{1}$ homotopy group is called a braid group. The configuration space for $N$ identical particles located on $M$ manifold (e.g., $R^{n}$ ) is defined as follows, $F_{N}(M)=M^{N} \backslash \Delta$, for distinguishable identical particles; $M^{N}$ is a $N$-fold normal product of $M$ manifold, $\Delta$ is a set of diagonal points (where the coordinates of two or more particles coincide), which has to be subtracted in order to conserve the number of particles in the system (particles cannot hide one behind another). The braid group is the first homotopy group [51,54] $\pi_{1}$ of the configuration space of a system of $N$ particles. Braid groups represent topologically different available trajectories of a system of $N$ particles (not referred to any specific dynamics). The pure braid group which we will utilize to neuron web is thus defined as

$$
\pi_{1}\left(F_{N}(M)\right)=\pi_{1}\left(M^{N} \backslash \Delta\right) .
$$

When trajectories (represented as the neuron filaments) are mutually entangled in the multi-line braid form, the initial ordering of particles must be unchanged. By $l_{i j}$ the elementary generators of the pure braid group can be assigned (Figure 12) [49], they correspond to the simplest entanglement of two trajectories of a particle pair, $i$-th and $j$-th, while the rest of the particle trajectories remain not-tangled. The generators $l_{i j}$ can be expressed with the use of $\sigma_{i}$ (exchanges of neighboring particles $i$-th and $i+1$-th not conserving their positions [49]),

$$
l_{i j}=\sigma_{j-1} \cdot \sigma_{j-2} \ldots \sigma_{i+1} \cdot \sigma_{i}^{2} \cdot \sigma_{i+1}^{-1} \ldots \sigma_{j-2}^{-1} \cdot \sigma_{j-1}^{-1}, \quad 1 \geq i \geq j \geq N-1 .
$$

Pure braid group can be considered as an abstract mathematical group generated by $l_{i j}$ defined by the following relations [49,52] (cf. Figure 13),

$$
l_{r s}^{-1} \cdot l_{i j} \cdot l_{r s}= \begin{cases}l_{i j,} & i<r<s<j \\ l_{i j}, & r<s<i<j \\ l_{r j} \cdot l_{i j} \cdot l_{r j}^{-1}, & r<i=s<j \\ l_{r j} \cdot l_{s j} \cdot l_{i j} \cdot l_{s j}^{-1} \cdot l_{r j}^{-1}, & i=r<s<j \\ l_{r j} \cdot l_{s j} \cdot l_{r j}^{-1} \cdot l_{s j}^{-1} \cdot l_{i j} \cdot l_{s j} \cdot l_{r j} \cdot l_{s j}^{-1} \cdot l_{r j}^{-1}, & r<i<s<j .\end{cases}
$$




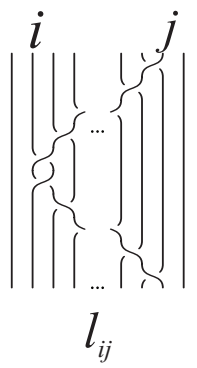

a)

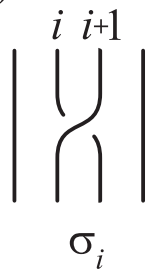

b)

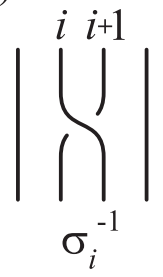

c)

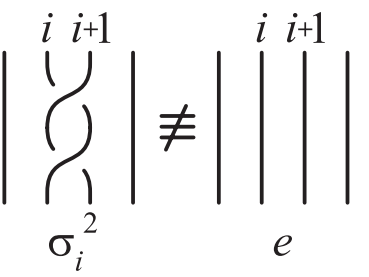

Figure 12. Generator $l_{i j}$ of the pure braid group (left), the exchange of neighboring particles, $\sigma_{i}(\mathbf{a})$ and its inverse, $\sigma_{i}^{-1}(\mathbf{b})$ and the reason why the braids on a plane are complicated (c).

a)

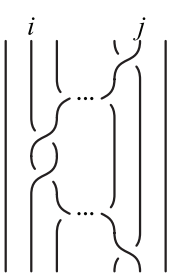

b)

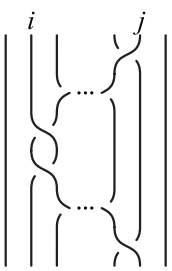

c)

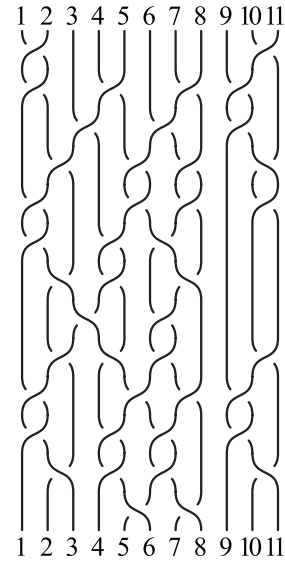

d)

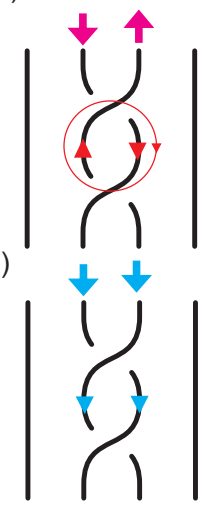

when ion current is pushed in opposite directions through two tangled threads then the scroll of an electrical coil is created

when ion current is pushed in same directions through two tangled threads then the scroll is not active

Figure 13. Generator $l_{i j}(\mathbf{a})$ and its inverse $l_{i j}^{-1}(\mathbf{b})$ of the pure braid group; an example of $N=11$ pure braid (c); the scheme of creating of an EM active (d) (non-active (e)) scroll of coil by entanglement of threads in a pure braid.

One can propose to identify a fragment of the neuron web connected via active synapses predefined by a prior record of some information in filament web as some pattern from the pure braid group. In particular, one can use the binary code to record in a three-filament bundle of any message (two generators of the corresponding $N=3$ pure braid group serve to code 0 and 1 bits and the third generator encodes the end of a word). It is possible to code of $N$-letter alphabet message in $\mathrm{N}$-order pure braid group [53]. We have demonstrated that the ratio of an information efficiency of $\mathrm{N}$-order pure braid group to resources needed to organize a physical web (like a neuron web with $N$ entangled filaments) attains its maximum at $N=20-30$, which agrees with the number of phones in majority languages [52]. This might suggest that words are codded in relatively small number of filaments in entangled bunches.

In the braid scenario we propose to code information in entanglement patterns in pieces of the neuron web in the brain selected by activation of some synapses. This is a topological nonlocal type of coding and is more efficient that the conventional addressing of synapse registers $(N$ synapses addressing offers only $N$ ! various connections with another $N$ synapse register, whereas the number of distinct brads of threads linking this registers is infinite, limited only by a physical constraint of finite size (diameter and length) of threads).

To identify neuron braids one can propose the EM resonance utilizing ion currents which flow in neuron filaments of the gray matter. Different roles are played by the white matter with axon cords corrugated by the periodic myelin sheath. The effective and fast saltatory transduction of the action potential through long myelinated axons of the peripheral nerves and in the white matter of the central nervous system undeniably stands behind the motorics and other communication functions of the body and requires at least 100 times faster signaling than that one offered by diffusion-type ion flows upon the cable theory. Actually such a high-speed signaling in myelinated axons is observed and 
called the saltatory conduction of action potential [13]. In myelinated axons, the action potential occurs in so-called Ranvier nodes [9] and is renewing on subsequent series of these nodes upon a very well recognized mechanism of Hodgkin Huxley membrane model [9]. However, in-between the Ranvier nodes along the myelinated sectors of an axon the signal hops, which accelerates strongly the action signal transduction along the periodically myelinated axon structure. The velocity of this saltatory conduction is at least two order faster than the diffusion ion current. Such an acceleration is absolutely required for proper functionality of long distance signaling in the peripheral nervous system but also for quick communication in the brain and in spinal cord in their white matter. It is interesting that in the brain the white matter is located in inner structures beneath the cortex, but in the spinal cord inversely. This might be linked with a different role of the gray matter in the cerebral cortex and in the spinal cord-the cortex is the place of memory storage and of it comparison or identification with new messages from surroundings accessing a brain via senses, whereas the gray matter in the spinal cord serves to identification of internal body information necessary to the control of body functioning. The latter must be precise and error-less thus the gray matter in a spinal cord ought to be isolated from surroundings as best as possible, here by an outer white matter layer. It has been demonstrated that by an EM external field, especially its magnetic component, one can influence and trans-cranially perturb identification process in the brain cortex, what would be, however, very danger in the spinal cord-thus the latter must be better isolated than the cortex in brain.

The white matter takes part thus in communication but is not utilized to recognition of braids with stored information which is the role of the gray matter. Actually the signal transduction in myelinated axons is not of electrical current character and thus cannot take part in EM identification of braids.

Such a scenario is consistent with the plasmon-wave type character of the saltatory conduction in myelinated axons as presented above. The saltatory conduction is apparently of a wave type because it is maintained even if a myelinated axon is divided into two disjoint pieces with ends separated slightly by an insulating barrier impossible to be crossed by any diffusive ion current.

We have provided the model for the saltatory conduction based on the kinetic properties of collective plasmon-polariton modes propagating along linear and periodically myelinated electrolyte system, which in the case of axon is the thin cord of the nerve cell periodically wrapped by Schwann cells creating thick myelin sheath (Schwann cells myelinate axons in the peripheral system, whereas in the central neural system the white matter is built from axons myelinated by the oligodendrocyte cells). Plasmon-polaritons were previously investigated and understood based on the very well developed nano-plasmonics in metal-chains $[15,16]$. It was demonstrated a long range low-damped propagation of plasmon-polaritons along metallic nano-chains [17]. Let us recall the main properties of these collective excitations occurring in metallic nano-chains on the conductor/insulator interface $[18,19]$ by hybridization of surface plasmons (i.e., fluctuations of charge density of electron fluid on the metal surface) with the electro-magnetic wave:

1. Much lower velocity of plasmon-polaritons in comparison to the light velocity, which gives much shorter wavelength of plasmon-polaritons than light wavelength with the same frequency, this causes a strong discrepancy of momenta of plasmon-polaritons and photons with the same energy; thus, plasmon-polaritons do not interact with EM waves, i.e., photons cannot be excited or absorbed by plasmon-polaritons,

2. Radiative losses of plasmon-polaritons do not exist and plasmon-polariton attenuation is only due to Ohmic losses, which makes metallic nano-chains almost perfect waveguides for plasmon-polaritons,

3. Long range and practically undamped propagation of plasmon-polaritons is observed experimentally $[17,20]$.

Some of the unique properties of plasmon-polaritons in metallic nano-chains are of especial interest as they can be repeated in periodic linear alignments of electrolyte systems with ions instead of electrons as charged carriers. Due to larger mass of ions in comparison to electrons and lower concentration of ions in electrolytes than the concentration of electrons in metals, plasmon resonances 
in finite ionic systems (e.g., in liquid electrolyte confined to the finite volume by appropriately formed membranes frequent in biological cell organization) occur in a different than in metals scale of micrometers instead of nanometers and for several orders lower frequency or energy (depending on ion concentration).

\section{Conclusions}

For spherical electrolyte micro-system one deals with the surface and volume plasmons in analogy to metallic nanosphere as demonstrated in the previous paragraph. The ionic surface plasmon frequencies are given for $l$-th multipole mode by the formula $\omega_{l}=\omega_{p} \sqrt{\frac{l}{\varepsilon(2 l+1)}}$, where the bulk plasmon frequency $\omega_{p}=\sqrt{\frac{4 \pi q^{2} n}{m}}$ ( $n$ is ion concentration, $q$ and $m$ are ion charge and mass, respectively, $\varepsilon$ is the dielectric relative permittivity of the surroundings). For dipole-type surface plasmons $(l=1)$ the frequency is given by so-called Mie-type formula, [1,6] $\omega_{1}=\frac{\omega_{p}}{\sqrt{3 \varepsilon}}$.

In the linear chain of ionic systems the irradiation losses are completely reduced to zero, exactly in the same manner as in metallic nano-chains [25-27]. The large irradiation energy losses expressed by the Lorentz friction at each chain element $[8,22]$ are ideally compensated by the income of energy due to radiation of the chain rest. In the result the radiative losses are ideally balanced by mutual radiation exchange and there remains only relatively small Ohmic irreversible energy dissipation. The collective plasmon-polaritons can thus propagate in the ionic chain with strongly reduced damping as in almost perfect wave-guide. If additionally the energy is supplemented in a minor scale to compensate small Ohmic losses, the propagation can reach arbitrarily long distances without any damping. This is what is observed in saltatory conduction in myelinated axons, moreover, plasmon-polaritons in this axons cannot be neither detected not perturbed by external EM field.

Advantages of plasmon-polariton kinetics in agreement with observed myelinated neuron signaling properties can be listed as follows:

- Plasmon-polaritons cannot be neither perturbed nor detected by EM waves (EEG signals are related to grey matter activity with ordinary conduction [according to the cable theory] and producing weak EM field, whereas myelinated white matter does not produce EEG signal).

- Immunity with respect to EM perturbation is convenient in long links in myelinated neurons of peripheral nervous system (motorics and sensing cannot be disturbed by EM field).

- All the EM field of plasmon-polaritons is compressed along the tight tunnel around the axon cord.

- The myelin sheath must be sufficiently thick not for the insulation reason but for creation of the dielectric tunnel in surrounding inter-cellular electrolyte to allow plasmon-polariton formation (reducing in myelin sheath thickness perturbs formation of plasmon-polaritons or lowers plasmon-polariton group velocity, resulting in Multiple Sclerosis).

- Increase of the temperature causes enhancement of Ohmic losses but does not reduce the plasmon-polariton group velocity (however, more energy ADP / ATP, residual to action potential spike formation, is needed to compensate the temperature increase of Ohmic losses).

- Any radiative losses do not occur for plasmon-polaritons; if plasmon-polariton is residually aided by spike formation (renovation) at consecutive Ranvier nodes, the arbitrary long range plasmon-polariton kinetics is possible with constant amplitude of the dipole signal.

- The plasmon-polariton group velocity increases with the cross-section of the axon cord.

- The plasmon-polariton group velocity for real neuron electrolyte and size parameters fits to the observed velocity of the saltatory conduction.

- Wave type of plasmon-polariton kinetics explains the maintenance of the signal transduction despite of small breaks and gaps in neuron cord or damage of some Ranvier nodes (these observations were impossible to be explained either within the cable theory or by compression-soliton model).

- One-direction kinetics if started from one end of the axon but two-direction kinetics if plasmon-polariton is ignited from some central point of an axon. 
Taking into account the above listed properties of plasmon-polariton conduction one might thus propose the nonlocal-topological of braid-group-type approach to information processing in brain taking into account the specific and different electricity of the gray and white matter of neurons. The web of neuron filaments in gray matter would serve to encode the information in the entanglement of filaments selected by activation of some synapses, because electricity of dendrites and of non-myelinated axons (in the gray matter) is of electrical current type providing the EM signature when AC signal is transducted trough the neuron network. The method of EM resonance identification of information stored in braided segments of the neuron web can be thus suggested. This method favors the gray matter with the ordinary ion currents of diffusive type (upon the cable model) but excludes the myelinated white matter with wave-type plasmon-polariton signaling without any EM signature. The saltatory conduction in myelinated axons in the white matter is not active with respect to EM resonance, as typical for plasmon-polaritons. We discussed this in detail and proposed the model of ionic plasmon-polariton propagation to explain the saltatory conduction in myelinated axons. This wave type propagation of the action potential is actually passive with respect to EM response and cannot contribute to the braid information processing in the gray matter, though conveniently fulfills communication role in the peripheral and central nervous systems. Plasmon-polariton model leads to an explanation of the efficient, very fast and long range so-called saltatory conduction in myelinated axons in peripheral neural systems and in white matter of the brain and spinal cord, where the velocity and energy-efficiency of communication is of the most significance. The coincidence with observations supports the propriety of the proposed new model for the saltatory conduction of the action potential, which, on the other hand, complementary fits to the different role and functioning of the gray matter. Both myelinated and non-myelinated structures share thus between them the different but complementary roles in the whole neuron system.

Author Contributions: Both co-authors contributed equally to the present paper.

Funding: The paper publication has been supported by K6 WUST.

Conflicts of Interest: The authors declare no conflict of interest.

\section{Appendix A. Derivation of Plasmon Frequencies for Finite Electrolyte System}

\section{Appendix A.1. Volume Ionic Plasmons}

In order to determine self-frequencies of the volume ionic plasmon in the sphere, we must solve Equation (18) with the form of the relevant solution given by Equation (20). For initial conditions listed below Equation (20) we assume $F(\mathbf{r}, t)=F_{\omega}(\mathbf{r}) \sin (\omega t)$ and by substitution of this function into Equation (18) we get,

$$
\Delta F_{\omega}(\mathbf{r})+k^{2} F_{\omega}(\mathbf{r})=0,
$$

where $k^{2}=\frac{\left(\omega^{2}-\omega_{p}^{2}\right) m}{k_{B} T}$. This is a well-known Helmholtz differential equation of which solutions (finite in origin) can be expressed by the spherical Bessel functions (for the radial dependence of $F_{\omega}(\mathbf{r})$ ),

$$
F_{\omega}(\mathbf{r})=A j_{l}(k r) Y_{l m}(\Omega),
$$

where $j_{l}(x)=\sqrt{\frac{\pi}{2 x}} I_{l+1 / 2}(x)$ is the the $l$-th spherical Bessel function linked to the Bessel function of first kind. The boundary condition, $F(a)=0$, gives quantization of $k, k_{l i}=\frac{x_{l i}}{a}$, where $x_{l i}$ is the $i$-th zero of the $l$-th Bessel function (cf. Figure 2 left). Following this quantization one arrives at the corresponding self-frequency quantization,

$$
\omega_{l i}^{2}=\omega_{p}^{2}\left(1+\frac{k_{B} T x_{l i}}{\omega_{p}^{2} m a^{2}}\right)
$$


Thus the volume ionic plasmons in the sphere are described by functions,

$$
\delta \rho_{1}(\mathbf{r}, t)=n \sum_{l=1}^{\infty} \sum_{m=-l}^{m=l} \sum_{i=1}^{\infty} A_{l m i} j_{l}\left(k_{l i} r\right) Y_{l m}(\Omega) \sin \left(\omega_{l i} t\right)
$$

where $A_{l m i}$ are arbitrary constants. The component with $l=0$ vanishes because of neutrality condition, $\int_{0}^{a} r^{2} d r d \Omega F(\mathbf{r}, t)=0\left(\right.$ as $\left.\int d \Omega Y_{l m}(\Omega)=\sqrt{4 \pi} \delta_{l 0} \delta_{m 0}, d \Omega=\sin \Theta d \Theta d \phi\right)$. Note that in ionic systems self-frequencies of volume plasmons in the sphere are temperature dependent-cf. Equation (A3) and Figure 2 right.

\section{Appendix A.2. Surface Ionic Plasmons}

In order to determine self-frequencies for surface plasmons, one has to consider Equation (19) and the solution for it given by Equation (20).

The first term in r.h.s. of Equation (19) can be rewritten to the form,

$$
\begin{aligned}
& \frac{k T}{m} \nabla\left(n+\delta \rho_{2}\right) \nabla \Theta(a-r)+\frac{k_{B} T}{m}\left(n+\delta \rho_{2}\right) \Delta \Theta \\
& =-\frac{k_{B} T}{m} \delta(a-r) \frac{\partial}{\partial r}\left(n+\delta \rho_{2}\right)-\frac{k_{B} T}{m}\left(n+\delta \rho_{2}\right) \frac{1}{r^{2}} \frac{\partial}{\partial r}\left(r^{2} \delta(a-r)\right) \\
& =-\frac{k_{B} T}{m} \frac{1}{r^{2}} \frac{\partial}{\partial r}\left[\left(n+\delta \rho_{2}\right) r^{2} \delta(a-r)\right]
\end{aligned}
$$

where we have used the formulae $\nabla \Theta(a-r)=-\frac{\mathrm{r}}{r} \delta(a-r), \frac{\mathrm{r}}{r} \nabla=\frac{\partial}{\partial r}$. The next term in r.h.s. of Equation (19) can be transformed into,

$$
\begin{aligned}
& -\frac{k_{B} T}{m} \delta(a-r) \frac{\mathbf{r}}{r} \nabla \delta \rho_{2}-\frac{\omega_{p}^{2}}{4 \pi} \delta(a-r) \frac{\mathbf{r}}{r} \nabla \int \frac{d^{3} r r_{1} \delta \rho\left(\mathbf{r}_{1}\right)}{\left|\mathbf{r}-\mathbf{r}_{1}\right|} \\
& =-\frac{k_{B} T}{m} \delta(a-r) \frac{\partial}{\partial r} \delta \rho_{2}-\frac{\omega_{p}^{2}}{4 \pi} \delta(a-r) \frac{\partial}{\partial r} \int \frac{d^{3} r_{1} \delta \rho\left(\mathbf{r}_{1}\right)}{\left|\mathbf{r}-\mathbf{r}_{1}\right|} .
\end{aligned}
$$

Equation (19) attains thus the form,

$$
\begin{aligned}
& \frac{\partial^{2} \rho_{2}}{\partial t^{2}}=-\frac{k_{B} T}{m} \frac{1}{r^{2}} \frac{\partial}{\partial r}\left[\left(n+\delta \rho_{2}\right) r^{2} \delta(a-r)\right] \\
& -\frac{k_{B} T}{m} \delta(a-r) \frac{\partial}{\partial r} \delta \rho_{2}-\frac{\omega_{p}^{2}}{4 \pi} \delta(a-r) \frac{\partial}{\partial r} \int \frac{d^{3} r_{1} \delta \rho\left(\mathbf{r}_{1}\right)}{\left|\mathbf{r}-\mathbf{r}_{1}\right|} .
\end{aligned}
$$

We suppose the solution of the above equation in the form, $\delta \rho_{2}=\sigma(\Omega, t) \delta\left(a+0^{+}-r\right)$ and multiply both sides of this equation by $r^{2}$ and integrate with respect to $r$ in arbitrary limits, i.e., $\int_{l}^{L} r^{2} d r \ldots$, such that $a \in(l, L)$ (this integration removes Dirac deltas), which leads to the equation,

$$
\begin{aligned}
& a^{2} \frac{\partial^{2} \sigma(\Omega, t)}{\partial t^{2}}=-\frac{k_{B} T}{m} \int_{l}^{L} d r \frac{\partial}{\partial r}\left[\left(n+\delta \rho_{2}\right) r^{2} \delta(a-r)\right] \\
& -\frac{k_{B} T}{m} \sigma(\Omega, t) \int_{l}^{L} r^{2} d r \delta(a-r) \frac{\partial}{\partial r} \delta(a-r) \\
& -\frac{\omega_{p}^{2}}{4 \pi} \int_{l}^{L} r^{2} d r \delta(a-r) \frac{\partial}{\partial r} \int_{a}^{\infty} r_{1}^{2} d r_{1} \int d \Omega \frac{\delta \rho_{2}\left(\mathbf{r}_{2}\right)}{\left|\mathbf{r}-\mathbf{r}_{1}\right|} \\
& -\frac{\omega_{p}^{2}}{4 \pi} \int_{l}^{L} r^{2} d r \delta(a-r) \frac{\partial}{\partial r} \int_{0}^{a} r_{1}^{2} d r_{1} \int d \Omega \frac{\delta \rho_{1}\left(\mathbf{r}_{1}\right)}{\left|\mathbf{r}-\mathbf{r}_{1}\right|}
\end{aligned}
$$

Two first terms in r.h.s. of the above equation vanish, because,

$$
-\frac{k_{B} T}{m} \int_{l}^{L} d r \frac{\partial}{\partial r}\left[\left(n+\delta \rho_{2}\right) r^{2} \delta(a-r)\right]=-\left.\frac{k_{B} T}{m}\left[\left(n+\delta \rho_{2}\right) r^{2} \delta(r-a)\right]\right|_{l} ^{L}=0
$$


and

$$
\begin{aligned}
& \int_{l}^{L} r^{2} d r \delta(a-r) \frac{\partial}{\partial r} \delta(a-r)=a^{2} \int_{l}^{L} d r \frac{1}{2} \frac{\partial}{\partial r} \delta^{2}(a-r) \\
& =\left.\frac{a^{2}}{2} \delta^{2}(a-r)\right|_{l} ^{L}=\left.\frac{a^{2}}{2} \lim _{\mu \rightarrow 0} \frac{1}{\pi} \frac{\mu}{\mu^{2}+(a-r)^{2}} \delta(a-r)\right|_{l} ^{L}=0 .
\end{aligned}
$$

Two last terms of r.h.s of Equation (A8) can be transformed using the formula [39], $\frac{1}{\sqrt{1+z^{2}-2 z \cos \gamma}}=$ $\sum_{l=0}^{\infty} P_{l}(\cos \gamma) z^{l}$, for $z<1$, where $P_{l}(\cos \gamma)=\frac{4 \pi}{2 l+1} \sum_{m=-l}^{l} Y_{l m}(\Omega) Y_{l m}^{*}(\Omega)$ are Legendre polynomials. This formula leads to the following one,

$$
\frac{\partial}{\partial a} \frac{1}{\left|\mathbf{a}-\mathbf{r}_{1}\right|}=\left\{\begin{array}{l}
\sum_{l=0}^{\infty} \frac{l a^{l-1}}{r_{1}^{l+1}} P_{l}(\cos \gamma), \text { for } a<r_{1} \\
-\sum_{l=0}^{\infty} \frac{(l+1) r_{1}^{l}}{a^{l+2}} P_{l}(\cos \gamma), \text { for } a>r_{1}
\end{array}\right.
$$

where $\mathbf{a}=a \frac{\mathbf{r}}{r}, \cos \gamma=\frac{\mathbf{a} \cdot \mathbf{r}_{1}}{a r_{1}}$. Employing Equation (A11) the last two terms in Equation (A8) can be transformed as follows,

$$
\begin{aligned}
& -\frac{\omega_{p}^{2}}{4 \pi} \int_{l}^{L} r^{2} d r \delta(a-r) \frac{\partial}{\partial r} \int_{a}^{\infty} r_{1}^{2} d r_{1} \int d \Omega_{1} \frac{\delta \rho_{2}\left(\mathbf{r}_{1}\right)}{\left|\mathbf{r}-\mathbf{r}_{1}\right|} \\
& =-\frac{\omega_{p}^{2}}{4 \pi} a^{2} \int d \Omega_{1} \int_{a}^{\infty} r_{1}^{2} d r_{1} \delta \rho_{2}\left(\mathbf{r}_{1}\right) \frac{\partial}{\partial a} \frac{1}{\sqrt{a^{2}+r_{1}^{2}-2 a r_{1} \cos \gamma}} \\
& =-\frac{\omega_{p}^{2}}{4 \pi} a^{2} \int d \Omega_{1} \int_{a}^{\infty} r_{1}^{2} d r_{1} \sigma\left(\Omega_{1}\right) \delta\left(a+0+-r_{1}\right) \sum_{l=0}^{\infty} \frac{l a^{l-1}}{r_{1}^{l+1}} P_{l}(\cos \gamma) \\
& =-\frac{\omega_{p}^{2}}{4 \pi} a^{2} \int d \Omega_{1} \sigma\left(\Omega_{1}\right) \frac{1}{a^{2}} \sum_{l=0}^{\infty} \frac{4 \pi l}{2 l+1} \sum_{m=-l}^{l} Y_{l m}(\Omega) Y_{l m}^{*}\left(\Omega_{1}\right) \\
& =-\omega_{p}^{2} a^{2} \sum_{l=0}^{\infty} \sum_{m=-l}^{l} \frac{l}{2 l+1} Y_{l m}(\Omega) \int d \Omega_{1} \sigma\left(\Omega_{1}\right) Y_{l m}^{*}\left(\Omega_{1}\right)
\end{aligned}
$$

and

$$
\begin{aligned}
& -\frac{\omega_{p}^{2}}{4 \pi} \int_{l}^{L} r^{2} d r \delta(a-r) \frac{\partial}{\partial r} \int_{0}^{a} r_{1}^{2} d r_{1} \int d \Omega_{1} \frac{\delta \rho_{1}\left(\mathbf{r}_{1}\right)}{\left|\mathbf{r}-\mathbf{r}_{1}\right|} \\
& =-\frac{\omega_{p}^{2}}{4 \pi} a^{2} \int d \Omega_{1} \int_{0}^{a} r_{1}^{2} d r_{1} n F\left(\mathbf{r}_{1}, t\right)\left(\mathbf{r}_{1}\right) \frac{\partial}{\partial a} \frac{1}{\sqrt{a^{2}+r_{1}^{2}-2 a r_{1} \cos \gamma}} \\
& =\frac{\omega_{p}^{2}}{4 \pi} a^{2} \int d \Omega_{1} n F\left(\mathbf{r}_{1}, t\right) \sum_{l=0}^{\infty} \frac{(l+1) r_{1}^{l}}{a^{l+2}} P_{l}(\cos \gamma) \\
& =\omega_{p}^{2} n \sum_{l=0}^{\infty} \frac{l+1}{2 l+1} Y_{l m}(\Omega) \int_{0}^{a} r_{1}^{2} d r_{1} \frac{r_{1}^{l}}{a^{l}} \sum_{l_{1}=1}^{\infty} \sum_{m_{1}=-l_{1}}^{l_{1}} \\
& \sum_{i} A_{l m i} j_{l_{1}}\left(k_{l_{1}} r_{1}\right) \sin \left(\omega_{l_{1} i} t\right) \int d \Omega_{1} Y_{l m}^{*}\left(\Omega_{1}\right) Y_{l_{1} m_{1}}\left(\Omega_{1}\right) \\
& =\omega_{p}^{2} n \sum_{l=0}^{\infty} \sum_{m=-l}^{l} \sum_{i} \frac{l+1}{2 l+1} Y_{l m}(\Omega) A_{l m i} \int_{0}^{a} \frac{r_{1}^{l+2} d r_{1}}{a^{l}} j_{l}\left(k_{l i} r_{1}\right) \sin \left(\omega_{l i} t\right) .
\end{aligned}
$$

Equation (A8) attains thus the form,

$$
\begin{aligned}
& \frac{\partial^{2} \sigma(\Omega, t)}{\partial t^{2}}=-\omega_{p}^{2} a^{2} \sum_{l=0}^{\infty} \sum_{m=-l}^{l} \frac{l}{2 l+1} Y_{l m}(\Omega) \int d \Omega_{1} \sigma\left(\Omega_{1}\right) Y_{l m}^{*}\left(\Omega_{1}\right) \\
& +\omega_{p}^{2} n \sum_{l=0}^{\infty} \sum_{m=-l}^{l} \sum_{i} \frac{l+1}{2 l+1} Y_{l m}(\Omega) A_{l m i} \int_{0}^{a} \frac{r_{1}^{l+2} d r_{1}}{a^{l}} j_{l}\left(k_{l i} r_{1}\right) \sin \left(\omega_{l i} t\right),
\end{aligned}
$$


Assuming now, $\sigma(\Omega, t)=\sum_{l=0}^{\infty} \sum_{m=-l}^{l} q_{l m}(t) Y_{l m}(\Omega)$ and putting it to the above equation, we obtain,

$$
\begin{aligned}
& \sum_{l=0}^{\infty} \sum_{m=-l}^{l} Y_{l m}(\Omega) \frac{\partial^{2} q_{l m}(t)}{\partial t^{2}}=-\sum_{l=0}^{\infty} \sum_{m=-l}^{l} \frac{\omega_{p}^{2} l}{2 l+1} Y_{l m}(\Omega) q_{l m}(t) \\
& +\omega_{p}^{2} \sum_{l=1}^{\infty} \sum_{m=-l}^{l} \sum_{i} \frac{l+1}{2 l+1} Y_{l m}(\Omega) A_{l m} \int_{0}^{a} \frac{r_{1}^{l+2} d r_{1}}{a^{l+2}} j_{l}\left(k_{l i} r_{1}\right) \sin \left(\omega_{l i} t\right) .
\end{aligned}
$$

From the above equation we notice that for $l=0$ we get $\frac{\partial^{2} q_{00}}{\partial t^{2}}=0$ and thus $q_{00}(t)=0$ (as $q(0)=0$ and $\left.\lim _{t \rightarrow \infty} q(t)<\infty\right)$. For $l \geq 1$ we get,

$$
\frac{\partial^{2} q_{l m}(t)}{\partial t^{2}}=-\frac{\omega_{p}^{2} l}{2 l+1} q_{l m}(t)+\sum_{i} \omega_{p}^{2} \frac{l+1}{2 l+1} A_{l m} n \int_{0}^{a} \frac{r_{1}^{l+2} d r_{1}}{a^{l+2}} j_{l}\left(k_{l i} r_{1}\right) \sin \left(\omega_{l i} t\right),
$$

which induces the solution form as follows,

$$
\begin{aligned}
& q_{l m}(t)=B_{l m} / a^{2} \sin \left(\omega_{p} \sqrt{\frac{l}{2 l+1}} t\right) \\
& +\sum_{i} A_{l m} \frac{(l+1) \omega_{p}^{2}}{\omega_{p}^{2}-(2 l+1) \omega_{l i}^{2}} n \int_{0}^{a} \frac{r_{1}^{l+2} d r_{1}}{a^{l+2}} j_{l}\left(k_{l i} r_{1}\right) \sin \left(\omega_{l i} t\right)
\end{aligned}
$$

and $\delta \rho_{2}(\mathbf{r}, t)=\sum_{l=1}^{\infty} \sum_{m=-l}^{l} q_{l m}(t) Y_{l m}(\Omega) \delta(a-r)$.

The first term in Equation (A17) describes self-frequencies of surface plasmons, whereas the second one displays the surface plasmon oscillations induced by the volume plasmons. This induced part of surface oscillations is nonzero only when the volume modes are excited and their amplitudes, $A_{l m i}$, are nonzero. The frequencies of self-oscillations of the surface plasmons are equaled to $\omega_{l 0}=$ $\omega_{p} \sqrt{\frac{l}{2 l+1}}$, corresponding to various multipole modes (numbered with $l$ ). Note that these frequencies are lower that the bulk plasmon frequency $\left(\omega_{p}=\sqrt{\frac{n q^{2} 4 \pi}{m}}\right.$ in Gauss units or $\sqrt{\frac{n q^{2}}{\varepsilon_{0} m}}$ in SI), whereas the volume plasmon modes oscillate with frequencies higher than $\omega_{p}$. Worth noting is also the absence of the temperature dependence of surface ionic plasmon resonances in contrary to volume plasmon self-frequencies.

Concluding, we can state that in ionic finite systems we may observe plasmons similar as in the metallic nanoparticles. The structure of surface and volume plasmons for ions is repeated from the similar properties of electronic plasmons in metallic spherical systems, however, with the significant shift of resonant energy towards lower one correspondingly to by few orders larger mass of ions in comparison to electron mass and different concentration of ions in electrolyte. Thus corresponding to the resonant energy electro-magnetic wavelength is shifted to deep infra-red or even longer wavelengths depending on ion concentration. The typical for metal clusters cross-over in size dependence of plasmon damping between the scattering, leading to Ohmic-type energy dissipation, versus the irradiation losses also is observable in ionic spherical system with similar to metal size-dependence, though shifted toward the micrometer scale for ions instead of the nanometer scale for metals. Of particular interest is the high irradiation regime for dipole plasmons in ionic system with prospective application for signaling and energy transfer in ionic systems. The initial strong enhancement of efficiency of the Lorentz friction with the radius growth of electrolyte sphere is observed on the micrometer scale with typical $a^{3}$ radius dependence above some threshold radius which value depends on electrolyte parameters. At certain value of the radius (variating in a wide range also depending on ion system parameters) this enhancement saturates and then slowly diminishes, which allows for definition of the most convenient sizes of electrolyte finite system for optimizing radiation mediated transport efficiency. 


\section{References}

1. Jacak, J.; Krasnyj, J.; Jacak, W.; Gonczarek, R.; Chepok, A.; Jacak, L. Surface and volume plasmons in metallic nanospheres in semiclassical RPA-type approach; near-field coupling of surface plasmons with semiconductor substrate. Phys. Rev. B 2010, 82, 035418. [CrossRef]

2. Pines, D. Elementary Excitations in Solids; ABP Perseus Books: Boston, MA, USA, 1999.

3. Brack, M. The physics of simple metal clusters: Self-consistent jellium model and semiclassical approaches. Rev. Mod. Phys. 1993, 65, 667. [CrossRef]

4. Ekardt, W. Size-dependent photoabsorption and photoemission of small metal particles. Phys. Rev. B 1985, 31, 6360. [CrossRef]

5. Jacak, W.; Krasnyj, J.; Jacak, J.; Gonczarek, R.; Chepok, A.; Jacak, L.; Hu, D.; Schaadt, D. Radius dependent shift of surface plasmon frequency in large metallic nanospheres: Theory and experiment. J. Appl. Phys. 2010, 107, 124317. [CrossRef]

6. Mie, G. Beitrige zur Optik trüber Medien, speziell kolloidaler Metallösungen. Ann. Phys. 1908, 25, 376.

7. Brongersma, M.L.; Hartman, J.W.; Atwater, H.A. Electromagnetic energy transfer and switching in nanoparticle chain arrays below the diffraction limit. Phys. Rev. B 2000, 62, R16356. [CrossRef]

8. Landau, L.D.; Lifshitz, E.M. Field Theory; Nauka: Moscow, Russia, 1973.

9. Debanne, D.; Campanac, E.; Białowąs, A.; Carlier, E.; Alcaraz, G. Axon physiology. Physiol. Rev. 2011, $91,555$. [CrossRef]

10. Thomson, W. On the theory of the electric telegraph. Proc. R. Soc. Lond. 1854, 7, 382-399.

11. Lazarevich, I.A.; Kazantsev, V.B. Dendritic signal transmission induced by intracellular charge inhomogeneities. Phys. Rev. E. 2013, 88, 062718. [CrossRef]

12. Brzychczy, S.; Poznañski, R. Mathematical Neuroscience; Academic Press: San Diego, CA, USA, 2011.

13. Lillie, R.S. Factors affecting transmission and recovery in passive iron nerve model. J. Gen. Physiol. 1925, 7, 473-507. [CrossRef]

14. Heimburg, T.; Jackson, A.D. On soliton propagation in biomembranes and nerves. Proc. Natl. Acad. Sci. USA 2005, 102, 9790-9795. [CrossRef] [PubMed]

15. Barnes, W.L.; Dereux, A.; Ebbesen, T.W. Surface plasmon subwavelenght optics. Nature 2003, 424, 824-830. [CrossRef] [PubMed]

16. Pitarke, J.M.; Silkin, V.M.; Chulkov, E.V.; Echenique, P.M. Theory of surface plasmons and surface-plasmon polaritons. Rep. Progr. Phys. 2007, 70, 1-87. [CrossRef]

17. Maier, S.A.; Atwater, H.A. Plasmonics: Localization and guiding of electromagnetic energy in metal/dielectric structures. J. Appl. Phys. 2005, 98, 011101. [CrossRef]

18. Zayats, A.V.; Smolyaninov, I.I.; Maradudin, A.A. Nano-optics of surface plasmon polaritons. Phys. Rep. 2005, 408, 131-314. [CrossRef]

19. de Abajo, F.J.G. Optical excitations in electron microscopy. Rev. Mod. Phys. 2010, 82, 209. [CrossRef]

20. Maier, S.A.; Kik, P.G.; Atwater, H.A. Optical pulse propagation in metal nanoparticle chain waveguides. Phys. Rev. B 2003, 67, 205402. [CrossRef]

21. Citrin, D.S. Plasmon Polaritons in Finite-Length Metal-Nanoparticle Chains: The Role of Chain Length Unravelled. Nano Lett. 2005, 5, 985-989. [CrossRef]

22. Jackson, J.D. Classical Electrodynamics; John Willey and Sons Inc.: New York, NY, USA, 1998.

23. Jacak, W. Plasmons in finite spherical electrolyte systems: RPA effective jellium model for ionic plasma excitations. Plasmonics 2015. [CrossRef]

24. Jacak, W. Size-dependence of the Lorentz friction for surface plasmons in metallic nanospheres. Opt. Exp. 2015, 23, 4472-4481. [CrossRef]

25. Citrin, D. Plasmon-polariton transport in metal-nanoparticle chains embedded in a gain medium. Opt. Lett. 2006, 31, 98-100. [CrossRef]

26. Markel, V.A.; Sarychev, A.K. Propagation of surface plasmons in ordered and disordered chains of metal nanospheres. Phys. Rev. B 2007, 75, 085426. [CrossRef]

27. Jacak, W.A. On Plasmon Polariton Propagation Along Metallic Nano-Chain. Plasmonics 2013, 8, 1317-1333. [CrossRef] 
28. Jacak, W. Propagation of Collective Surface Plasmons in Linear Periodic Ionic Structures: Plasmon Polariton Mechanism of Saltatory Conduction in Axons. J. Phys. Chem. C 2015, 119, 10015. [CrossRef]

29. Zhao, L.L.; Kelly, K.L.; Schatz, G.C. The Extinction Spectra of Silver Nanoparticle Arrays: Influence of Array Structure on Plasmon Resonance Wavelength and Width. J. Phys. Chem. B 2003, 107, 7343-7350. [CrossRef]

30. Zou, S.; Janel, N.; Schatz, G.C. Silver nanoparticle array structures that produce remarkably narrow plasmon lineshapes. J. Chem. Phys. 2004, 120, 10871. [CrossRef] [PubMed]

31. Singham, S.B.; Bohren, C.F. Light scattering by an arbitrary particle: A physical reformulation of the coupled dipole method. Opt. Lett. 1987, 12, 10-12. [CrossRef] [PubMed]

32. Jensen, T.; Kelly, L.; Lazarides, A.; Schatz, G.C. Electrodynamics of Noble Metal Nanoparticles and Nanoparticle Clusters. J. Clust. Sci. 1999, 10, 295-317. [CrossRef]

33. Draine, B.T. The discrete-dipole approximation and its application to interstellar graphite grains. Astrophys. J. 1988, 333, 848-872. [CrossRef]

34. Purcell, E.M.; Pennypacker, C.R. Scattering and absorption of light by nanospherical dielectric grains. Astrophys. J. 1973, 186, 705-714. [CrossRef]

35. Draine, B.T.; Flatau, P.J. Discrete-dipole approximation for scattering calculations. J. Opt. Soc. Am. A 1994, 11,1491-1499. [CrossRef]

36. Markel, V.A. Coupled-dipole approach to scattering of light from a onedimensional periodic dipole structure. J. Mod. Opt. 1993, 40, 2281-2291. [CrossRef]

37. Maier, S.A.; Kik, P.G.; Sweatlock, L.A.; Atwater, H.A.; Penninkhof, J.J.; Polman, A.; Meltzer, S.; Harel, E.; Requicha, A.; Koel, B.E. Energy transport in metal nanoparticle plasmon waveguides. Mat. Res. Soc. Symp. Proc. 2003, 777, T7.1.1.

38. Jacak, W.; Krasnyj, J.; Chepok, A. Plasmon-Polariton Properties in Metallic Nanosphere Chains. Materials 2015, 8, 3910-3937. [CrossRef]

39. Gradshteyn, I.S.; Ryzhik, I.M. Table of Integrals Series and Products; Academic Press, Inc.: Boston, MA, USA, 1994.

40. Jacak, W. Exact solution for velocity of plasmo-polariton in metallic nano-chain. Opt. Expr. 2014, 22, 18958-18965. [CrossRef]

41. Citrin, D. Coherent excitation transport in metal-nanoparticle chains. Nano Lett. 2004, 4, 1561-1565. [CrossRef]

42. Agranovich, V.M.; Dubovskii, O.A. Effect of retarded interaction on the exciton spectrum in one-dimensional and two-dimensional crystals. JETP Lett. 1966, 3, 223.

43. Meissner, T.; Wentz, F. The Complex Dielectric Constant of Pure and Sea Water from Microwave Satellite Observations. IEEE THRS 2004, 42, 1836-1849. [CrossRef]

44. El-Brolossy, T.A.; Abdallah, T.; Mohamed, M.B.; Abdallah, S.; Easawi, K.; Negm, S.; Talaat, H. Shape and size dependence of the surface plasmon resonance of gold nanoparticles studied by Photoacoustic technique. Eur. Phys. J. Spec. Top. 2008, 153, 361-364. [CrossRef]

45. Liz-Marzan, L.M. Tuning nanorod surface plasmon resonances. SPIE Newsroom 2007. [CrossRef]

46. Goubau, G. Surface Waves and Their Application to Transmission Lines. J. Appl. Phys. 1950, 21, 1119-1128. [CrossRef]

47. Sommerfeld, A. Über die fortpflanzung elektrodynamischer Wellen langs eines Drahts. Ann. Phys. Chem. 1899, 67, 233-290. [CrossRef]

48. Andrianov, E.; Pukhov, A.A.; Dorofeenko, A.V.; Vinogradov, A.P.; Lisyansky, A.A. Stationary behavior of a chain of interacting spasers. Phys. Rev. B 2012, 85, 165419. [CrossRef]

49. Birman, J.S. Braids, Links and Mapping Class Groups; Princeton UP: Princeton, NJ, USA, 1974.

50. Luka, G.; Krajewski, T.A.; Witkowski, B.S.; Wisz, G.; Virt, I.S.; Guziewicz, E.; Godlewski, M. Aluminum-doped zinc oxide films grown by atomic layer deposition for transparent electrode applications. J. Mater. Sci. Mater. Electron. 2011, 22, 1810-1815. [CrossRef]

51. Mermin, D. The topological theory of defects in ordered media. Rev. Mod. Phys. 1979, 51, 591. [CrossRef]

52. Jacak, J.; Gonczarek, R.; Jacak, L.; Jóźwiak, I. Application of Braid Groups in 2D Hall System Physics; World Scientific: Singapore, 2012. 
53. Stokowski, H.; Jacak, J. Multi-character alphabet coding using braid group formalism. Comput. Math. Appl. submitted.

54. Spanier, E. Algebraic Topology; Springer VL: Berlin, Germany, 1966.

(c)

(C) 2019 by the authors. Licensee MDPI, Basel, Switzerland. This article is an open access article distributed under the terms and conditions of the Creative Commons Attribution (CC BY) license (http:/ / creativecommons.org/licenses/by/4.0/). 